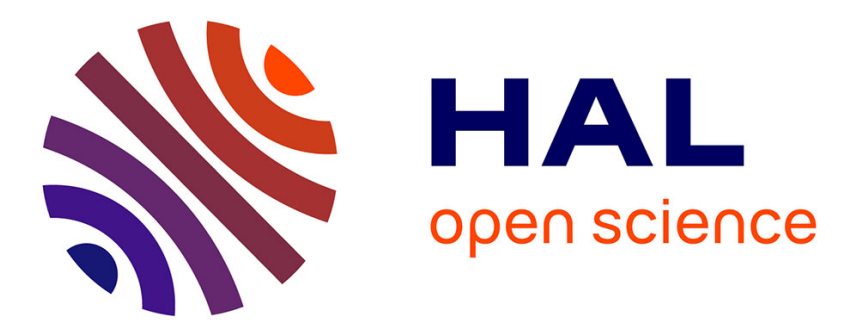

\title{
DO-CRIME: Dynamic On-sky Covariance Random Interaction Matrix Evaluation, a novel method for calibrating adaptive optics systems
}

\author{
Olivier Lai, Mark Chun, Ryan Dungee, Jessica Lu, Marcel Carbillet
}

\section{To cite this version:}

Olivier Lai, Mark Chun, Ryan Dungee, Jessica Lu, Marcel Carbillet. DO-CRIME: Dynamic On-sky Covariance Random Interaction Matrix Evaluation, a novel method for calibrating adaptive optics systems. Monthly Notices of the Royal Astronomical Society, 2020, 10.1093/mnras/staa3874 . hal$03037127 \mathrm{v} 2$

\section{HAL Id: hal-03037127 \\ https://hal.science/hal-03037127v2}

Submitted on 7 Dec 2020

HAL is a multi-disciplinary open access archive for the deposit and dissemination of scientific research documents, whether they are published or not. The documents may come from teaching and research institutions in France or abroad, or from public or private research centers.
L'archive ouverte pluridisciplinaire HAL, est destinée au dépôt et à la diffusion de documents scientifiques de niveau recherche, publiés ou non, émanant des établissements d'enseignement et de recherche français ou étrangers, des laboratoires publics ou privés. 


\title{
DO-CRIME: Dynamic On-sky Covariance Random Interaction Matrix Evaluation, a novel method for calibrating adaptive optics systems.
}

\author{
Olivier Lai ${ }^{1 \star}$, Mark Chun ${ }^{2}$, Ryan Dungee ${ }^{2}$, Jessica $\mathrm{Lu}^{3}$, and Marcel Carbillet ${ }^{1}$ \\ ${ }^{1}$ Université Côte d'Azur, Observatoire de la Côte d'Azur, CNRS, Laboratoire Lagrange, Bd de l'Observatoire, CS 34229, 06304 Nice cedex 4, France \\ ${ }^{2}$ Institute for Astronomy, University of Hawaii, 640 North A 'ohōkū Place, Hilo 96720, USA \\ ${ }^{3}$ Department of Astronomy, University of California at Berkeley, Berkeley CA 94720, USA
}

Accepted XXX. Received YYY; in original form ZZZ

\begin{abstract}
Adaptive optics systems require a calibration procedure to operate, whether in closed loop or even more importantly in forward control. This calibration usually takes the form of an interaction matrix and is a measure of the response on the wavefront sensor to wavefront corrector stimulus. If this matrix is sufficiently well conditioned, it can be inverted to produce a control matrix, which allows to compute the optimal commands to apply to the wavefront corrector for a given wavefront sensor measurement vector. Interaction matrices are usually measured by means of an artificial source at the entrance focus of the adaptive optics system; however, adaptive secondary mirrors on Cassegrain telescopes offer no such focus and the measurement of their interaction matrices becomes more challenging and needs to be done on-sky using a natural star. The most common method is to generate a theoretical or simulated interaction matrix and adjust it parametrically (for example, decenter, magnification, rotation) using on-sky measurements. We propose a novel method of measuring on-sky interaction matrices $a b$ initio from the telemetry stream of the AO system using random patterns on the deformable mirror with diagonal commands covariance matrices. The approach, being developed for the adaptive secondary mirror upgrade for the imaka wide-field AO system on the UH2.2m telescope project, is shown to work on-sky using the current imaka testbed.
\end{abstract}

Key words: atmospheric effects, techniques: high angular resolution, instrumentation: adaptive optics, high angular resolution, telescopes, methods: numerical, observational

\section{INTRODUCTION}

The foundational principle of adaptive optics (AO) is to measure a wavefront disturbance using a wavefront sensor (WFS) and to correct the measured aberrations in real time using a wavefront corrector, usually a deformable mirror (DM) but potentially, any phase inducing device (LCD, lenses misalignment, etc.) could be used (Babcock 1953). A computer is used to translate the wavefront sensor measurements into DM commands in real time. In effect the real time computer needs to solve a set of linear equations relating the measurement vector $\mathbf{m}$ to the commands vector $\mathbf{c}$.

$\mathbf{c}=A \cdot \mathbf{m}$

where $A$ is a matrix usually called the control matrix (Tyson 1991; Roddier 1999). At optical and infrared wavelengths, it is usually not possible to measure the phase of a light wave directly, so an optical device has to be used to transform a function of the phase into a mea-

$\star$ E-mail: olivier.laioca.eu surable intensity, which is why a non-trivial set of linear equations is needed to reconstruct the wavefront. For example, interferometric sensors transform the sine of the phase into intensity (usually leading to a limited linear range and a $2 \pi$ ambiguity); Shack-Hartman and pyramid wavefront sensors transform the gradient of the phase into an intensity centroid measurement and curvature sensors transform the laplacian of the phase into an intensity difference measurement. The advantage of the latter comes from their use with bimorph mirrors, which produce a constant curvature over their electrodes. In theory, the one-to-one correspondance between the measurements and the correction is equivalent to a perfectly diagonal matrix $A$. In practice, there is sufficient cross-talk in curvature systems to warrant a wavefront reconstructor such as the one described by equation 1 (i.e. with non-diagonal terms in $A$ ). All modern AO systems use some variant of Equation 1 in their control system and optimally mapping the actuator to the measurements is the topic of numerous studies.

The most common way to obtain the control matrix $A$ is to measure the so-called interaction matrix which we will call $D$. It is 
constructed by exciting each actuator or degree of freedom of the wavefront corrector sequentially and recording the wavefront sensor measurements such that:

$$
\mathbf{m}=D \cdot \mathbf{c}
$$

We might be tempted to write that $D=\mathbf{m} \cdot \mathbf{c}^{-1}$, but this equation doesn't capture the process of acquiring the interaction matrix by sequentially exciting linear combinations of commands or modes. Furthermore the inverse of a vector is not a well defined concept; although in the above equation, it can be thought of as $\mathbf{c}^{-1}=\mathbf{c}^{T} /|\mathbf{c}|^{2}$.

Instead, we concatenate each sequential command to write a matrix of commands $C$, where each column corresponds to an actuation of the deformable mirror. For example, sequentially exciting each actuator would make $C$ a diagonal matrix. Multiplying $D$ by $C$ produces a matrix of measurements $M$, in which each column corresponds to the response vector associated with command vector (the $n^{\text {th }}$ column of $C, \mathbf{c}_{i, n}$ ). We can then write:

$$
\begin{aligned}
M & =D \cdot C \\
D & =M \cdot C^{-1}
\end{aligned}
$$

At this point we note that nothing predetermines the matrix of commands $C$ to be diagonal, and it has been proposed that an optimal method of measuring interaction matrices consists in making $C$ a Hadamard matrix to generate the strongest signal, the strongest diversity of measurements on the wavefront sensor (Kasper et al. 2004; Meimon et al. 2015). However, since the command matrix $C$ matrix has to be inverted, there are advantages to it being diagonal (in which case it is simply $C^{-1}=C^{T} /|C|^{2}$ ). This method is what we will call the poke matrix in the following.

If $D$ is well conditioned then a general solution for $\mathrm{A}$ is given by the Moore-Penrose inverse $D^{+}$:

$A=D^{+}=\left(D^{T} \cdot D\right)^{-1} \cdot D^{T}$

However, in practice, $D$ is more often inverted with Singular Value Decomposition (SVD), which allows to filter eigenmodes of $D$ which have particularly low eigenvalues, e.g (Gendron \& Léna 1994; Lai et al. 2000); physically these are modes that are poorly sensed by the system, they produce a small measurement vector given a normalised stimulus. If not filtered, such modes are very sensitive to noise in the measurement vector and are amplified. Singular value decomposition is a generalisation of eigenmodes for non-square matrices using polar decomposition. We can write the interaction matrix $D$ as the product of three matrices $U, \Sigma$ and $V^{*}$ :

$D=U \cdot \Sigma \cdot V^{*}$

If $D$ is an $m \times n$ matrix (measurement vector has $m$ elements and DM has $n$ actuators), then $U$ is an $m \times m$ unitary matrix, $\Sigma$ is an $m \times n$ rectangular diagonal matrix with non-negative numbers on the diagonal and $V$ is also a unitary matrix, of dimension $n \times n$. As $D$ is a real matrix, $U$ and $V$ are real orthonormal matrices. The diagonal entries $\sigma_{i}=\Sigma_{i i}$ are known as the singular values of $D$. The number of non-zero singular values is equal to the number of independent degrees of freedom of the AO system. The pseudo-inverse of D is then given by:

$D^{+}=V \cdot \Sigma^{+} \cdot U^{*}$

where $\Sigma^{+}$is the pseudo-inverse of $\Sigma$ which is formed by replacing every non-zero diagonal entry by its reciprocal and transposing the resulting matrix. If values of $\Sigma_{i i}$ are very small, the corresponding value $\Sigma_{i i}^{+}$can be set to zero, thereby effectively filtering this particular mode, or degree of freedom from the reconstruction. We call the number of controlled modes $\Xi$, so it is a parameter in equation 7, $D^{+}(\Xi)=V \cdot \Sigma^{+}(\Xi) \cdot U^{*}$. Different AO geometries have such modes, called invisible modes or the null space of the control matrix: waffle mode in the case of Shack-Hartman wavefront sensors in a Fried geometry, or piston for curvature sensors.

Measuring the interaction matrix D usually requires an artificial source at the input focus of the AO system, which allows to record the wavefront sensor measurement vector while the wavefront corrector is actuated. However with the development of Adaptive Secondary Mirrors (ASM), it is not always possible to introduce a calibration source due to the lack of an intermediate or entrance focus. This is true for Cassegrain telescopes, but even for Gregorian telescopes, which do have an intermediate focus before reflection on the secondary mirror, this focus is not always easily accessible (Pieralli et al. 2008; Heritier et al. 2018). We also note that using an artificial source may not reflect the state of the system when it is in use, for example Shack-Hartman spots which would be diffraction limited with a calibration source may be blurred by atmospheric turbulence, or the telescope pupil may not match the AO system pupil exactly leading to a different illumination on the lenslet array. Finally, measuring an interaction matrix by "poking" each actuator and waiting for the system to settle will hide dynamical effects, such as dynamical coupling or variable time lag of actuators (which acts as a gain in closed loop). We therefore suggest that measuring interaction matrices on-sky and in the same conditions as during operation of the AO system has advantages beyond their use with ASMs.

Methods to generate interaction matrices when no intermediate focus is available fall into two major categories: Methods developed mostly for the ESO Adaptive Optics Facility (AOF) are what could be described as non-invasive as they generate a simulated or synthetic interaction matrix using models for the influence functions and the wavefront sensors and then use sky data to adjust alignment parameters such as the pupil translation, magnification and rotation on the wavefront sensor and/or the deformable mirror. Such methods have been investigated by Bechet et al. (2011, 2012), for the VLT AOF but also in light of the future ELT. The parametric fitting used on the AOF is described by Kolb et al. (2012) and Neichel et al. (2012) studied parametric adjustment of the misregistration for the SAXO AO system in the SPHERE high contrast instrument, the GeMS MCAO system and the HOMER wide field AO testbench. The parametric adjustment of the LBT pyramid wavefront misregistration has been studied by Heritier et al. (2018, 2019).

Another method to improve synthetic or simulated interaction matrices on-sky is more invasive, as it requires modulation of the deformable mirror (usually one or a few modes) and synchronous detection of these mode on the wavefront sensor. These methods have mostly been developed and used at LBT (Wildi \& Brusa. 2004; Oberti et al. 2006; Esposito et al. 2006). The relative merits of the invasive and non-invasive methods have been studied by Oberti et al. (2006) and more recently by Heritier et al. (2017) who do not reach a definite conclusion, each having advantages and drawbacks: the non-invasive approach obviously has no impact on observations, is fast and produces arbitrarily large SNR, but depends on complex models of the wavefront sensor, which may be an issue for the ELT, while the invasive method works well for small misregistrations but does have a (small) impact on observations and only a few modes can be measured at any time, so it is a slower process. Our method is slightly different since, although invasive, it is quite fast ( $\sim 10$ seconds, depending on the frame rate) to measure the entire interaction matrix, but unlike non-invasive methods, it is completely model independent, irrespective of the scale of the misregistration. 
Although the invasive and non-invasive methods work very well and are used extensively in practice, they have intrinsic limitations. For instance, the number of parameters to fit can become large if these are not global (e.g. pupil centering, magnification, rotation), and parameters can only fit known effects (see our example of malfunctioning electrode 10, Section 4.4 ). Synthetic matrices assume perfect lenslet arrays, but small defects (in focal length or pitch) can go unnoticed in parametric fits. The dynamic response of the deformable mirror may not be identical for every actuator, especially as a function of temperature as seen on magnetic actuators deformable mirrors, (Woillez et al. 2019), effectively acting as a differential gain on specific actuators. Edge subapertures also require specific care; their partial illumination leads to a lower signal to noise ratio, which can be mitigated by appropriate weighting. If the pupil moves with respect to the lenslet array during observations (for example due to flexures or pupil nutation), these weights need to be readjusted. This is especially critical if the centroiding algorithm uses non-linear filters such as thresholding, or if the guide source is extended. Finally, in the case of GLAO which is of particular interest to us, wavefront sensors can be far off-axis, leading to pupil distortions that can be hard to parametrize, e.g. if the illumination on the wavefront sensor becomes elliptical or vignetted or if the actuator pattern is distorted with respect to the lenslet array.

Therefore we suggest that measuring interaction matrices onsky ab-initio provides a more accurate measurement of the state of the AO system, without any assumptions or theoretical models. Interaction matrices contain all the information about the system alignment and gain response and we suggest that measuring them regularly is a useful diagnostic tool and may improve the quality of the correction, especially when very high performance is required. In cases where the deformable mirror rotates with respect to the lenslet array (e.g. adaptive secondary mirror with WFS at Nasmyth or Coudé focus), our method can be used to generate on-the-fly interaction matrices or to control a pupil derotator to stabilize the WFS-DM alignement. The amount of on-sky time required for nonscience calibration observations is low for this approach, and no modification is needed (e.g. introducing an artificial source), with the telescope and $\mathrm{AO}$ system at the same inclination and attitude (and hence flexures) as the science observations.

\section{METHOD}

In closed loop, a simple integrator control loop follows the following discrete time step equation:

$\mathbf{c}_{t+1}=\mathbf{c}_{t}+g A \cdot \mathbf{m}_{t}$

Where $g$ is a scalar loop gain (usually $<1$ ). Therefore, we can re-arrange and write:

$A \cdot \mathbf{m}_{t}=\frac{1}{g}\left(\mathbf{c}_{t+1}-\mathbf{c}_{t}\right)$

We alleviate the notation by writing $\Delta \mathbf{c}_{t}=\mathbf{c}_{t+1}-\mathbf{c}_{t}$. By multiplying both sides by $\mathbf{m}_{t}^{T}$ and rearranging:

$A=\frac{1}{g}<\Delta \mathbf{c}_{t} \cdot \mathbf{m}_{t}^{T}>\cdot<\left(\mathbf{m}_{t} \cdot \mathbf{m}_{t}^{T}\right)>^{-1}$

where $<>$ signfies the time average. Thus $<\left(\mathbf{m}_{t} \cdot \mathbf{m}_{t}^{T}\right)>$ is the measurements covariance matrix. Conversely from equations 2 and 9 , we can also write:

$g \mathbf{m}_{t}=D \Delta \mathbf{c}_{t}$
Multuplying both sides by $\Delta \mathbf{c}_{t}^{T}$, taking the time average and rearranging, we obtain:

$\left.D=g<\mathbf{m}_{t} \cdot \Delta \mathbf{c}_{t}^{T}\right\rangle \cdot\left\langle\Delta \mathbf{c}_{t} \cdot \Delta \mathbf{c}_{t}^{T}\right\rangle^{-1}$

$<\left(\Delta \mathbf{c}_{t} \cdot \Delta \mathbf{c}_{t}^{T}\right)>$ is the incremental commands covariance matrix. The control loop bandwidth of AO systems is usually higher than the atmospheric coherence time, so for atmospheric disturbances, the variance of the differences of subsequent commands, $\sigma_{\Delta \mathbf{c}}^{2}$, is small.

Our method consists in applying a random (but known) vector $\mathbf{c}_{\xi}(t)$ of commands and recording the measurements. This can be done in closed loop, but if the control matrix is not known on first pass, this can also be done in open loop, as long as the signal remains within the linearity range of the wavefront sensor (this can be an issue for curvature or unmodulated pyramid sensing or ShackHartman using quad-cells). On sky in open loop, equation 2 can be used to write:

$\mathbf{m}_{\xi}(t)+\mathbf{m}_{a}(t)=D \cdot \mathbf{c}_{\xi}(t)$

Where $\mathbf{m}_{a}(t)$ is the measurement of the atmosphere perturbation at instant $t$. We multiply both sides by $\mathbf{c}_{\xi}(t)^{T}$ and take the time average:

$<\mathbf{m}_{\xi}(t) \cdot \mathbf{c}_{\xi}(t)^{T}>+<\mathbf{m}_{a}(t) \cdot \mathbf{c}_{\xi}(t)^{T}>=D<\mathbf{c}_{\xi}(t) \cdot \mathbf{c}_{\xi}(t)^{T}>$

We measure $\mathbf{m}=\mathbf{m}_{\xi}+\mathbf{m}_{a}$ but on average there is no correlation between the atmosphere and our noise signal $\mathbf{c}_{\xi}(t)$, such that $<\mathbf{m}_{a}(t) \cdot \mathbf{c}_{\xi}(t)^{T}>\rightarrow 0$ and the interaction matrix is then given by:

$D=<\mathbf{m}_{\xi} \cdot \mathbf{c}_{\xi}^{T}>\cdot<\mathbf{c}_{\xi} \cdot \mathbf{c}_{\xi}^{T}>^{-1}$

In practice we can use the measurement $\mathbf{m}$ in equation 15 but we have to obtain sufficient number of realisations for the covariance of the atmosphere and the random commands to become small enough as to become negligible. However we can improve our estimate of $\mathbf{m}_{\xi}$ by frequentially separating and rejecting the contribution of the atmosphere $\mathbf{m}_{a}$, which will be strongest at low temporal frequencies in the measurements $\mathbf{m}$ by restricting our random signal to high frequencies, which we can achieve by applying a high pass filter to both $\mathbf{c}_{\xi}$ and $\mathbf{m}_{\xi}$. Therefore we set the amplitude of the random commands to be sufficiently large to produce a meaningful signal on the wavefront sensor, though it may still be smaller than the low frequency atmosphere disturbances: it can be fine-tuned depending on the application (e.g. real time acquisition during science exposure, e.g. see Section 3.2 and Fig. 7). The reason for using a random command vector is that the the covariance matrix $\left\langle\mathbf{c}_{\xi}(t) \cdot \mathbf{c}_{\xi}^{T}(t)>\right.$ (and for that matter $\left\langle\Delta \mathbf{c}_{\xi}(t) \cdot \Delta \mathbf{c}_{\xi}^{T}(t)>\right.$ in the closed loop case) will be diagonal and thus well conditioned for inversion.

If there are invisible or poorly sensed modes in the system, they can be filtered during the SVD inversion process. However, if the control matrix $A$ is obtained directly from equation 10 , the measurements covariance matrix $<\mathbf{m}(t) \cdot \mathbf{m}^{T}(t)>$ will not be well conditioned and will need filtering at inversion, achieving the same effect. We also note in passing that we tried to use slope Hadamard matrices for exciting the sensor response optimally as described by Meimon et al. (2015), but found no improvement in final accuracy with respect to using random vectors though our method uses more iterations due to the presence of turbulence that needs to be averaged out. We interpret this as whatever optimisation is gained in the Hadamard scheme is lost in the inversion of the commands (or measurements) covariance matrix inversion. The main reason why this method is robust is because the $\left\langle\mathbf{c}_{t} \cdot \mathbf{c}_{t}^{T}>\right.$ matrices are diagonal and can be inverted exactly. 

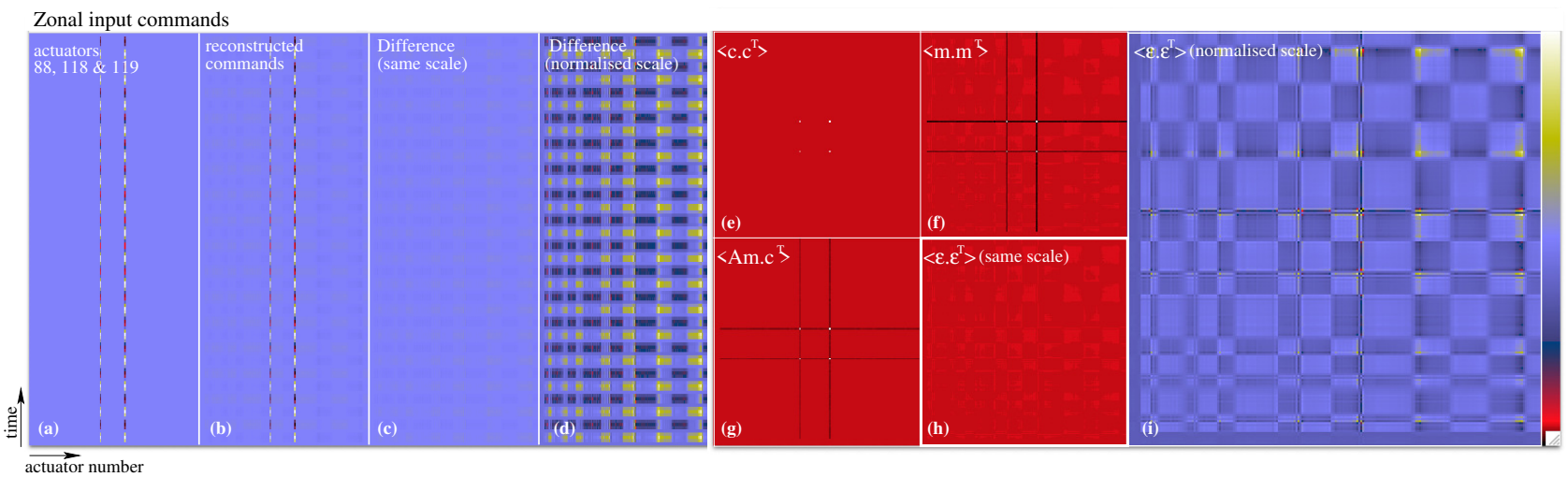

Figure 1. Zonal demonstration of the control matrix reconstruction fidelity simulation tool. Actuators 88,118 and 119 are excited sinusoidally (a), providing the input $\mathbf{c}_{a}$. Wavefront sensor measurements $\mathbf{m}_{a}$ are recorded and multiplied by the control matrix $A$ (b) and the difference $\epsilon_{a}$ (with the same color scale) is shown in (c); (d) shows this difference but stretched over the entire color scale. Panel (e) shows the covariance matrix of the commands, (f) shows the reconstructed commands, (g) is the cross term of equation 17 and (h) is the residual covariance matrix $\left\langle\epsilon \cdot \epsilon^{T}\right\rangle$. Panel (i) shows the residual covariance matrix stretched over the entire color scale. The trace of this matrix is the residual variance.
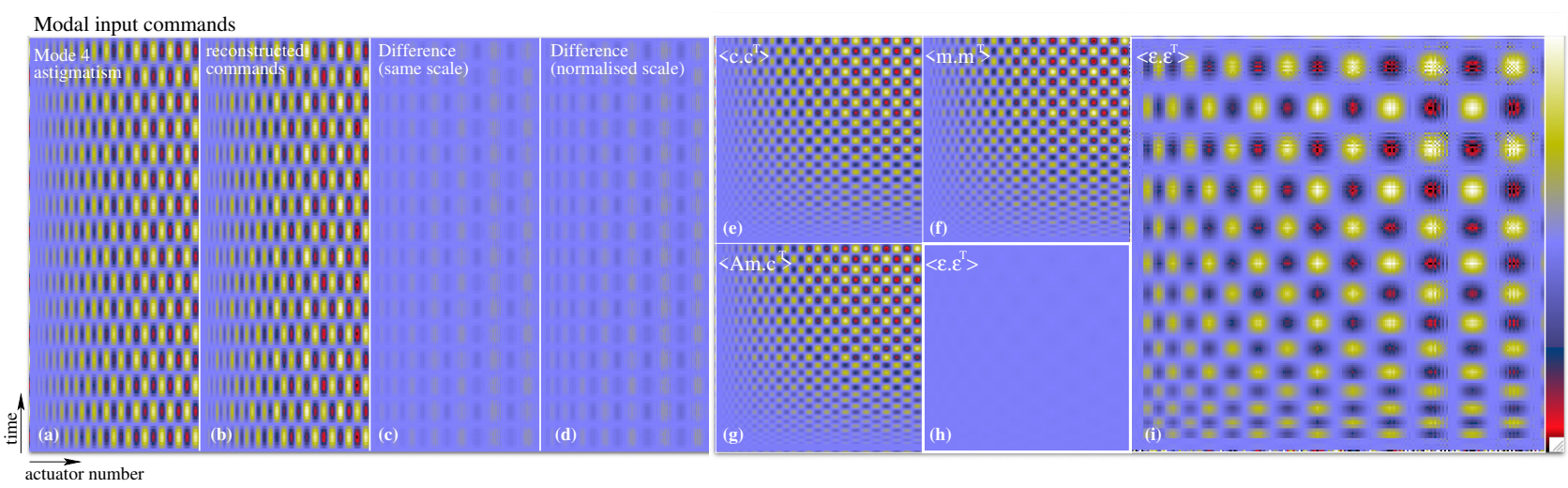

Figure 2. Modal (right) demonstration of the control matrix reconstruction fidelity simulation tool using mode 4, astigmatism. The panels are the same as in Fig. 1. Panels (e), (f), (g) and (h) show the four terms of equation 17.

\section{SIMULATIONS}

To test the DO-CRIME method we developed a simple simulation code based on a Shack Hartmann WFS model and the finite element model of the ASM influence functions; given any command vector $\mathbf{c}_{a}$, we can compute the corresponding response on the wavefront sensor, and generate the $\mathbf{m}_{a}$ measurement vector. We used realistic values for the dimensioning of the system: the 'imaka ASM is expected to have 211 actuators and we used a $16 \times 16$ WFS to sample them. With this tool, not only were we able to generate poke and DO-CRIME interaction matrices, but we were also able to assess the quality of the derived control matrices. This issue of how effective a control matrix actually is, contains two components: the first is the actual numerical conditioning of the matrix, meaning that a given matrix will propagate noise depending on its condition number. This is given by $\sigma_{\mathbf{c}}^{2}(\Xi)=\Sigma \operatorname{Trace}\left[D^{+^{T}}(\Xi) \cdot D^{+}(\Xi)\right]$. Physically, this noise propagation coefficient is the variance of the noise on the reconstructed commands when the input vector $\mathbf{m}$ contains $1 \mathrm{rad}^{2}$ of noise. The trivial case of this is that the noise propagation can be reduced by filtering out more modes, at the expense of accurate wavefront reconstruction. This leads us to develop a second quality criterion, namely the ability of the control matrix to reconstruct a wavefront for a given input vector. For example, if we modulate a given actuator at a known temporal frequency, and we measure the wavefront sensor vector, which we then multiply by the command matrix, how different is the reconstructed command vector to the input, how accurately does a given control matrix allow to reconstruct a command vector (Fig. 1, Fig. 10)? This will of course depend on the input commands and their cross-correlations. If the input commands generate a white noise command vector, then the noise propagation coefficient determines the quality of the matrix. But if the commands contain low spatial frequencies, which produce cross correlations across different areas of the pupil, different modes may need to be filtered; for example, if we modulate an astigmatism on the deformable mirror, a modal command matrix may be more effective than a zonal one (Fig. 2).

We call this quality criterion of control matrices the control matrix fidelity and we can quantify it by comparing the known input commands $\mathbf{c}_{a}$ with the measured output $D^{+}(\Xi) \cdot \mathbf{m}_{a}$ and measuring the residual difference $\epsilon_{a}(\Xi)$ :

$\epsilon_{a}(\Xi)=\mathbf{c}_{a}-D^{+}(\Xi) \cdot \mathbf{m}_{a}$

The optimal value of $\Xi_{o}$ (in other words, the optimal number of corrected modes) is the one that minimizes $\epsilon_{a}$ for which we define the optimal control matrix $A=D^{+}\left(\Xi_{o}\right)$. We can write the covariance 

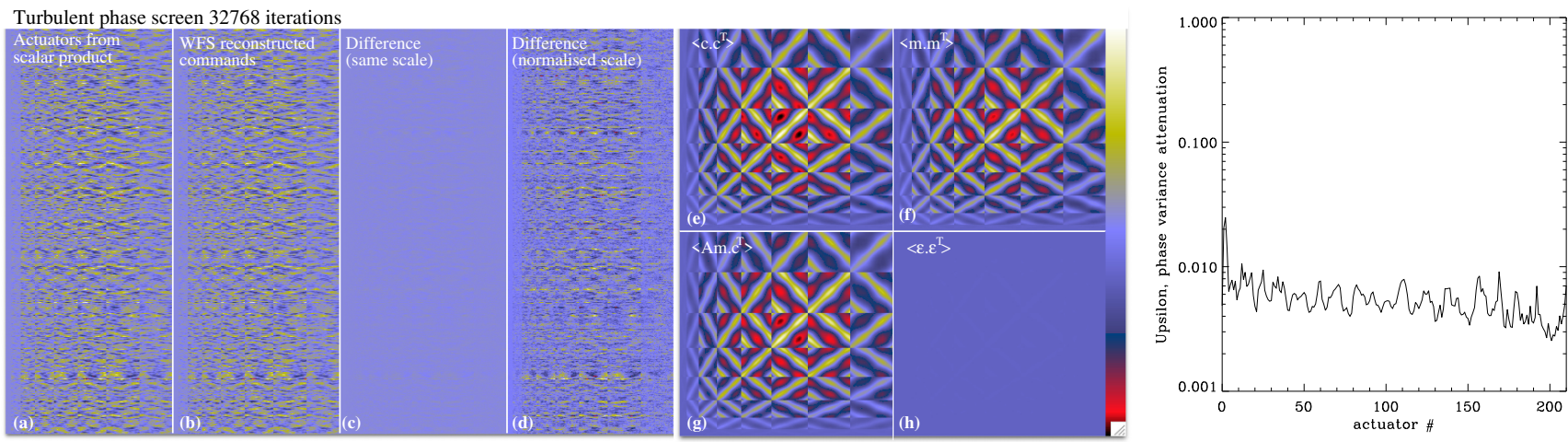

Figure 3. Same display as Fig. 1 but in phase space as opposed to actuator space, using turbulent phase screens to generate the commands (by scalar product with the influence functions) and the measurement through a model wavefront sensor (left). The covariance matrices of the commands $\left\langle\varsigma \cdot \varsigma^{T}>\right.$, of the measurements $\Delta_{i j} D^{+}<\mathbf{m} \cdot \mathbf{m}^{T}>D^{+T} \Delta_{i j}^{T}$ and of the cross terms $<\Delta_{i j} D^{+} \mathbf{m} \cdot \varsigma^{T}>$ are shown in the middle and are similar in overall structure. However, their residual difference $\left\langle\epsilon \cdot \epsilon^{T}>\right.$ (middle bottom right) is crucial as it is a measure of the control matrix ability to reconstruct turbulent wavefronts. The ratio of the trace of the residual phase covariance normalised to the input phase modulation, $\Upsilon_{\sigma_{\epsilon}^{2}}$ is shown for $\Xi=9$ on the right as a function of actuator number.

of the residual difference $<\epsilon_{a} \cdot \epsilon_{a}^{T}>$ as:

$$
\begin{aligned}
<\epsilon_{a} \cdot \epsilon_{a}^{T}>= & <\left(\mathbf{c}_{a}-D^{+} \cdot \mathbf{m}_{a}\right) \cdot\left(\mathbf{c}_{a}-D^{+} \cdot \mathbf{m}_{a}\right)^{T}> \\
= & <\mathbf{c}_{a} \cdot \mathbf{c}_{a}^{T}>+D^{+}<\mathbf{m}_{a} \cdot \mathbf{m}_{a}^{T}>D^{+^{T}}- \\
& <\mathbf{c}_{a} \cdot \mathbf{m}_{a}^{T} D^{+^{T}}+D^{+} \mathbf{m}_{a} \mathbf{c}_{a}^{T}> \\
= & <\mathbf{c}_{a} \cdot \mathbf{c}_{a}^{T}>+D^{+}<\mathbf{m}_{a} \cdot \mathbf{m}_{a}^{T}>D^{+^{T}}- \\
& <\left(\mathbf{c}_{a}\left(D^{+} \mathbf{m}_{a}\right)^{T}\right)+\left(D^{+} \mathbf{m}_{a} \mathbf{c}_{a}^{T}\right)>
\end{aligned}
$$

$<\mathbf{c}_{a} \cdot \mathbf{c}_{a}^{T}>$ is the statistical covariance matrix for which analytical expressions can be found in the literature (Gendron \& Léna 1994) and $<\mathbf{m}_{a} \cdot \mathbf{m}_{a}^{T}>$ is the slope covariance matrix for which it is also possible to compute analytical expressions based on the phase structure function (Lai et al. 2018). We also note that $\mathbf{c}_{a}\left(D^{+} \mathbf{m}_{a}\right)^{T}=\left(D^{+} \mathbf{m}_{a} \mathbf{c}_{a}^{T}\right)^{T}$, so the sum of the last two terms form a symmetric matrix. This equation tells us that the covariance of the residual of reconstruction are equal to the difference between the sum of the covariance of the commands and the covariance of the slopes (projected into command space) and the symmetric sum of their cross terms, as shown on panel (g) of Fig. 1, 2. There are no analytical expressions for these cross terms, as they contain the interaction between measurements and commands, specific to the adaptive optics system under consideration (i.e. actuator gains, misalignments, etc).

Nevertheless we can compute these matrices numerically using our simulation tool: we generate vectors of commands $\mathbf{c}_{a}$ according to the type of signal we want to reconstruct (in our case, Kolmogorov turbulence, but we can include noise or vibrations), and record their corresponding $\mathbf{m}_{a}$ responses. Then, for each control matrix of which we want to evaluate the fidelity, we compute $\epsilon_{a}$ according to Equation 16 from which we compute the residual covariance matrix $<\epsilon_{a} \cdot \epsilon_{a}^{T}>$. We can then compare sum of the traces of the different residual matrices associated with each control matrix. The commands $\mathbf{c}_{a}$, the reconstructed commands $\mathbf{m}_{a}$ and thus the residual $\epsilon_{a}$ are in actuator space, and the variance attenuation shown in Fig. 1, 2 is therefore on the commands applied to the deformable mirror. However, we are more interested in the phase variance attenuation. We can easily switch from actuator to phase space by multiplying $\mathbf{c}_{a}$ and $D^{+} \mathbf{m}_{a}$ by the geometrical covariance matrix (Gaffard \& Boyer 1987), defined as:

$$
\Delta_{i j}=\int_{\text {pupil }} M_{i}(\mathbf{r}) \cdot M_{j}(\mathbf{r}) d \mathbf{r}
$$

Where $M_{i}(\mathbf{r})$ and $M_{i}(\mathbf{r})$ are the $i$ and $j$ influence functions in units of phase over the pupil. In fact from our turbulence phase screen, we can obtain the phase coefficient $\varsigma_{a}$ directly by computing $\int_{\text {pupil }} \phi_{\text {turb }} M(\mathbf{r}) d \mathbf{r}$. We can then define the phase variance attenuation $\Upsilon_{\sigma_{\epsilon}^{2}}(\Xi)$ as the ratio of the phase variance of $\epsilon_{a}=\Delta_{i j} D^{+} \mathbf{m}_{a}$ to the input phase variance of the commands $S a$.

$\Upsilon_{\sigma_{\epsilon}^{2}}(\Xi)=\frac{\sum \operatorname{Tr}\left(<\epsilon_{a} \cdot \epsilon_{a}^{T}>\right)}{\sum \operatorname{Tr}\left(<\varsigma_{a} \cdot \varsigma_{a}^{T}>\right)}$

This phase variance attenuation is to be understood as the ability of the wavefront sensor and control matrix to reconstruct an input wavefront. As such it is a precise and robust prediction of open loop feed-forward adaptive optics. In closed loop however, the feedback correction introduces non-linear behaviour; some poorly conditioned modes can build up and make the loop unstable. We carried out extensive simulations and found that in closed loop, the optimal number of filtered modes can be different for the minimum phase variance attenuation and the best Strehl ratio, but without any clear or obvious pattern (the optimal number of filtered modes in closed loop is always greater or equal to the number of filtered modes for which $\Upsilon_{\sigma_{\epsilon}^{2}}$ is minimum). Qualitatively we see that the feedback loop can slowly build up invisible or poorly sensed modes which are not part of the interaction matrix basis (for example the island effect modes, or partial waffles). This effect can be (and usually is) mitigated by implementing a leaky integrator which adds further non-linearities. However, apart from running Monte Carlo simulations with varying number of filtered modes (which is particularly computer intensive), we have not found a reliable way to predict the optimal number of filtered modes in closed loop. Nonetheless, $\Upsilon_{\sigma_{\epsilon}^{2}}$ is a rigorous metric of wavefront reconstruction fidelity and is a good proxy for closed loop performance.

To test out the code we started with simple, synthetic signals: we applied zonal (actuating a few adjacent actuators with a sinusoidal modulation) to the ASM. We used a control matrix obtained from inverting a poke interaction matrix measured with the simulation tool. Sample results are displayed on Fig. 1 where 3 actuators are 


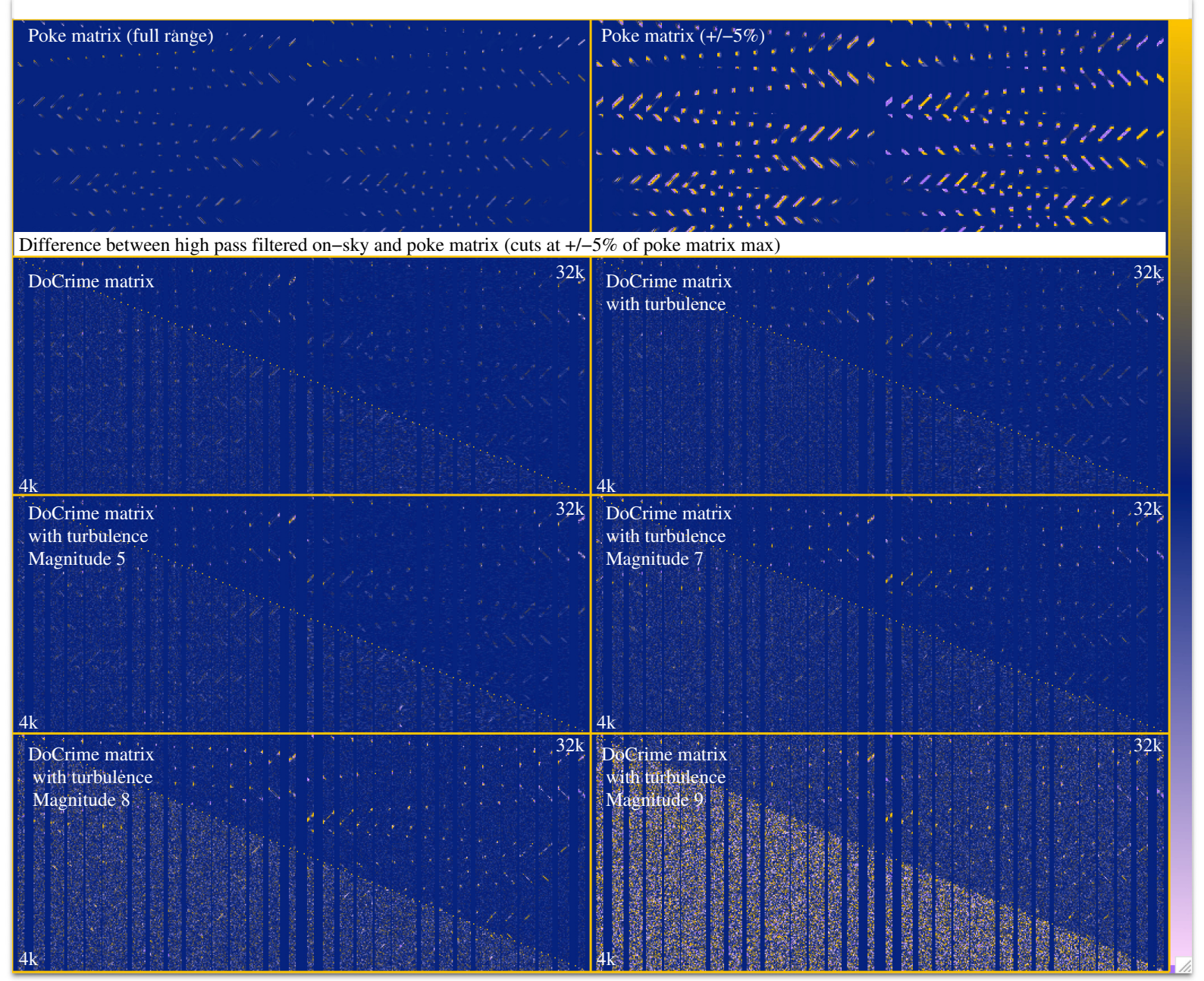

Figure 4. Top row, left: Poke interaction matrix, the shape of which depends on the geometry of the actuators and the subapertures. The radial pattern of actuators produces the oscillating pattern. Top right shows the same matrix with cuts at $\pm 5 \%$ of the maximum. The lower rows show the difference between the poke matrices and the interaction matrices obtained with the high pass filtered DO-CRIME method for the 6 different cases studied (no turbulence no noise, no noise but turbulence, and turbulence with increasing levels of noise). We show the differences between the six cases and the poke matrix for 4000 (bottom left half)and 32000 samples (top right half), with cuts at $\pm 5 \%$ of the maximum of poke matrix.

modulated sinusoidally. Modal modulation of the commands (modulating an astigmatism with a sinusoidal excitation coefficient) is shown on Fig. 2. In both cases, the control matrix is capable of accurately reconstructing the input signal and the epsilon covariance matrices show little or no structure; this is especially true in the case of the astigmatism, as the low order modes are better conditioned in control matrix (they have higher eigenvalues in the interaction matrix).

To be able to assess the fidelity of the reconstruction for real systems in real world situations, we need to determine the fidelity of control matrices to reconstruct atmospheric turbulence. We start with the open loop case: we simulate a phase screen and project it onto the deformable mirror to get a command vector (by computing the scalar product of the turbulent phase screen with the deformable mirror's influence functions as described previously). Simultaneously, we record the associated wavefront sensor measurement. We then generate a sequence of 32000 commands and associated measurements by sliding the turbulent phase screen across the pupil at are reasonable wind speed and $r_{0}$. We used the $\mathrm{UH} 2.2 \mathrm{~m}$ telescope entrance pupil with a $r_{0}$ of $0.2 \mathrm{~m}$, a windspeed of $8 \mathrm{~m} / \mathrm{s}$. These re- sults are shown on Fig. 3. Note that this figure is now in phase space, unlike Figs. 1, 2 which are in command space. We can see that the covariance matrices of the commands $\left\langle\varsigma \cdot \varsigma^{T}\right\rangle$, of the measurements $\Delta_{i j} D^{+}<\mathbf{m} \cdot \mathbf{m}^{T}>D^{+T} \Delta_{i j}^{T}$ and of the cross terms $<\Delta_{i j} D^{+} \mathbf{m} \cdot \mathbf{c}^{T}>$ are quite similar in overall structure, but their residual difference $<\epsilon \cdot \epsilon^{T}>$ is not zero and is a measure of the control matrix ability to reconstruct turbulent wavefronts. On this figure we also plot $\Upsilon_{\sigma_{\epsilon}^{2}}$ for $\Xi=9$ as a function of actuator number: the central actuators are not as well attenuated because they are partially hidden by the central obstruction; all other actuators attenuate the incoming phase variance by a factor $\sim 5 \times 10^{-3}$.

As our simulation tool now includes a sliding phase screen, we can also use it to generate DO-CRIME matrices in realistic onsky conditions, including atmospheric turbulence: we compute the shape of the deformable mirror associated with our random command coefficient vector $\mathbf{c}_{\xi}(t)$ and add it to the turbulence phase screen from which we generate a measurement vector (to which we can add noise or other realistic disturbances such as vibrations) from which we can generate interaction matrices according to Equation 15 . We can now study and optimize the input modulation for 

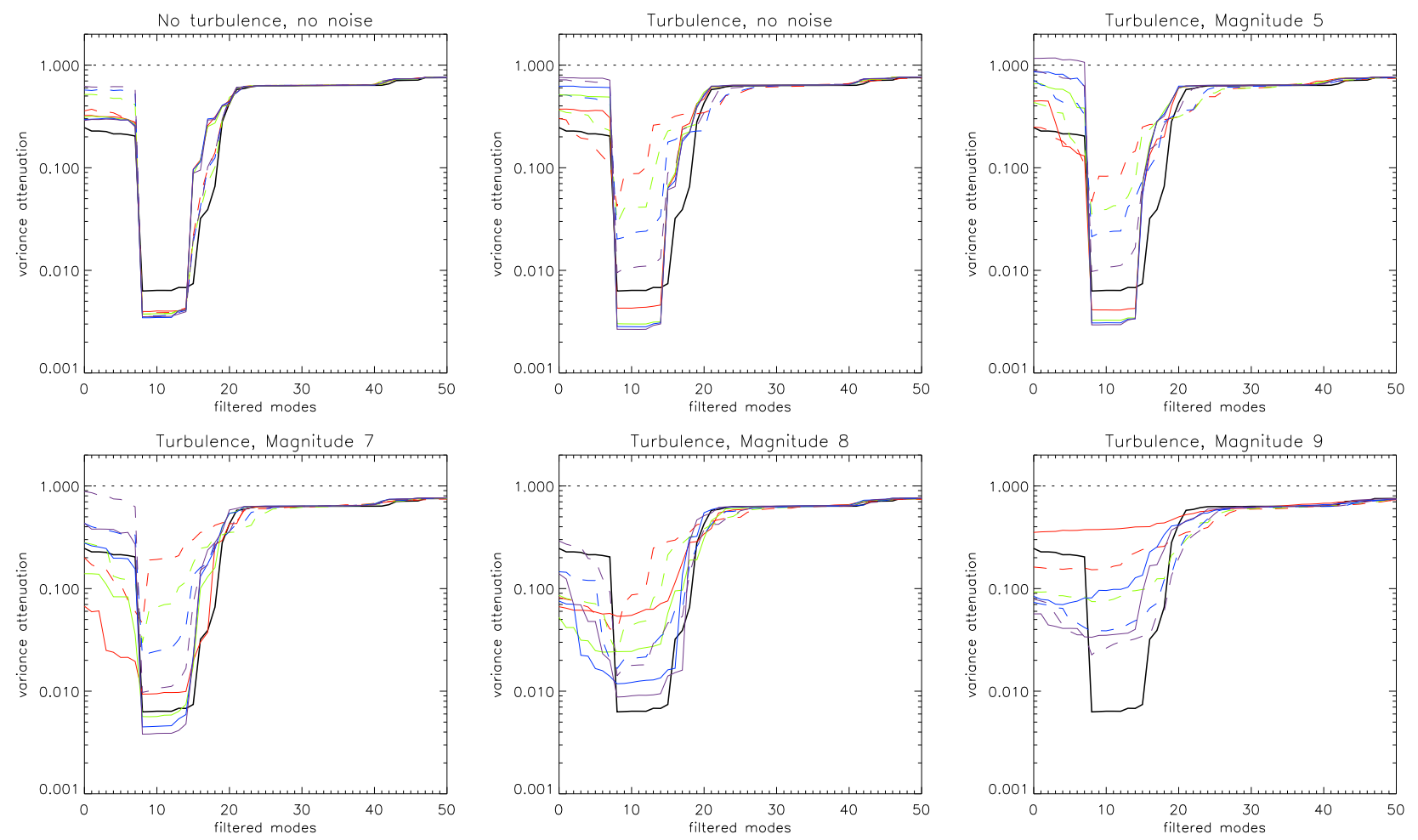

Figure 5. The attenuation of wavefront variance $\Upsilon_{\sigma_{\epsilon}^{2}}$ as a function of the number of filtered modes $\Xi$ during SVD inversion. When the number of filtered modes is too large, they asymptotically approach unity as less of the wavefront is corrected. When too few modes are corrected, invisible modes are included at inversion and make the loop unstable. The minimum (i.e. maximum attenuation) is around10 filtered modes (for this geometry). Black is the poke matrix, red is the interaction matrix obtained with 4000 iterations, green is with 8000 , blue 16000 and purple with 32000 iterations (at $600 \mathrm{~Hz}$, 7s, $14 \mathrm{~s}, 28 \mathrm{~s}$, and $56 \mathrm{~s}$ respectively). Full lines are high pass filtered data and full lines are the full data, containing the atmospheric signal. It is clearly apparent that the presence of turbulence can be well mitigated by the use of the high-pass filter, as can noise, as long as it doesn't dominate (up to magnitude 9 , equivalent to 200 detected photo-electrons per subaperture and per integration time, with $3 e^{-} /$pixel read noise and $12 \times 12$ pixels per subaperture)

the DO-CRIME method using these simulations, first in open loop, and then in closed loop to determine how many samples and what amplitude of modulation are needed so that these on-sky matrices can still be accurately measured in the presence of turbulence and noise.

\subsection{Open loop}

In open loop, we introduce a turbulent phase screen as well as noise on the wavefront sensor to study the control matrix fidelity as a function of number of iterations. With a sampling frequency set at $600 \mathrm{~Hz}$, sample sizes of 4096, 8192, 16384 and 32786, correspond to $6.8 \mathrm{~s}, 13.7 \mathrm{~s}, 27.3$ and $55 \mathrm{~s}$ respectively. We use a zero point of $9.6 \times$ $10^{10}$ detected photons/second over the entire pupil for a magnitude zero star, with stars of magnitudes 5, 7, 8 and 9. In more useful units, these correspond to 3300, 500, 200 and 80 detected photoelectrons per subaperture and per integration time. We also use $3 e^{-}$/pixel read noise and $12 \times 12$ pixels per subapterture. We then generate interaction matrices using the random $\mathbf{c}_{\xi}$ command vectors and the associated measurements $\mathbf{m}_{\xi}$ for the 6 following cases: No turbulence, no noise; turbulence (phase screen with $r_{0}=0.2 \mathrm{~m}$ ) but no noise; and turbulence with the 4 flux levels described above. We compute interaction matrices using the raw data as well as applying a high pass filter to better separate the turbulence from the random DO-CRIME signal, and display the results in Fig. 4. Qualitatively, the interaction matrices generated in almost all cases with 32000 samples are indistinguishable from the poke interaction matrix (apart from the highest noise case), and even with 4000 samples, the introduction of turbulence but no noise (or low noise level, magnitude 5) does not visually degrade the interaction matrix compared to the poke matrix.

To address this quantitatively, we invert these interaction matrices using Singular Value Decomposition, filtering from zero to 210 modes, and using our precomputed $\varsigma_{a}$ and $\mathbf{m}_{a}$ vectors, we compute the control matrix fidelity $\Upsilon_{\sigma_{\epsilon}^{2}}$ as a function of $\Xi$, which we plot for the 6 cases and the 4 number of iterations in Fig. 5.

We can point out a few salient features of these plots: The use of a high pass filter on the measurements allows to efficiently remove the contribution of the turbulence which is mainly at low frequency. If noise is negligible, then sequences with more than 8000 iterations (13 seconds @ 600Hz) are sufficient to measure interaction matrices that perform just as well as poke matrices, i.e. same minimum value of $\Upsilon_{\sigma_{\epsilon}^{2}}\left(\Xi_{o}\right)$. When the level of noise becomes more important, the fidelity of the control matrices can degrade substantially, and increasing the number of samples or iterations doesn't seem to be improve the resulting interaction matrices. This is most likely due to the inclusion of read noise and no thresholding in the centroid computation, as one would expect the results to degrade more gradually for photon noise only. Also, the fact that the noise is uncorrelated from one frame to the next also means its covariance matrix is diagonal and indistinguishable for the random commands sent on the DM, and high pass filtering cannot separate these contributions either. Thus the method works best with stars that are sufficiently bright, using a high pass filter on the data to 


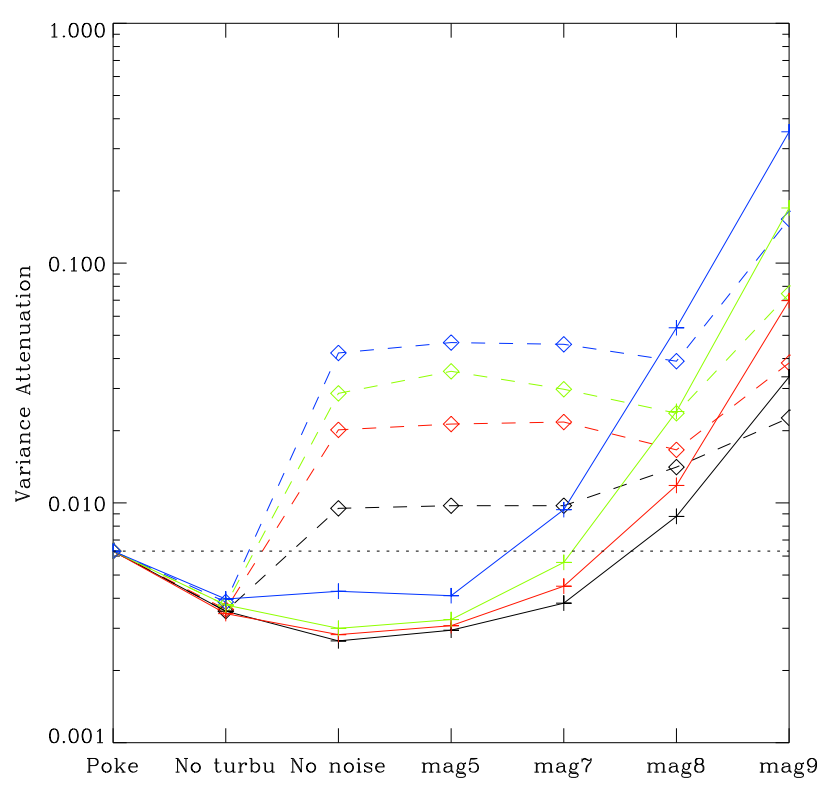

Figure 6. Minimum values of $\Upsilon_{\sigma_{\epsilon}^{2}}$ for each case, obtained by filtering the optimal number of modes $\Xi_{o}$ on Fig. 5. Form left to right: Poke matrix; no turbulence and no noise; turbulence and no noise, turbulence and magnitudes $5,9,10$ and 11 . Blue is the control matrix obtained with 4000 iterations, green is with 8000 , red 16000 and black with 32000 iterations, dashed lines are full data and full lines are high pass filtered.

remove most of the contribution of the turbulence, and to increase the amplitude of the random modulation to a level such that its random fluctuations are larger than the noise and thus dominate the signal on the wavefront sensor.

These results are summed up in Fig. 6, which shows the minimum value of $\Upsilon_{\sigma_{\epsilon}^{2}}\left(\Xi_{o}\right)$ obtained by filtering the optimal number of modes on Fig. 5.

When no turbulence and no noise is present, our matrices perform slightly better than the poke matrix because they naturally assign the optimal weight to partially illuminated edge subapertures (which is not done on the poke matrix), and 4000 iterations are sufficient to converge with or without high-pass filtering. The dashed lines show the results without temporal filtering and when turbulence is present, they do not perform as well as the poke matrix; for $4000,8000,16000$ and 32000 iterations, the phase variance attenuation goes from $4 \times 10^{-2}$ to $3 \times 10^{-2}$ to $2 \times 10^{-2}$ to $1 \times 10^{-2}$ respectively. From this we can infer that 64000 iterations may provide a comparable level of performance to the poke matrix but this is prohibitive to simulate and in fact not very useful on sky, as we can get much better results with a simple high pass filter on the data (full lines). The high pass filtered method works well in the presence of turbulence and noise, up to magnitude 7 (500ph/subaperture/integration time) when the number of sample is $>8000$, but $\Upsilon_{\sigma_{\epsilon}^{2}}\left(\Xi_{o}\right)$ starts to degrade for magnitude $8(200 \mathrm{ph} /$ subaperture/integration time) and higher.

\subsection{Closed loop simulations}

Since our simulation tool allows to record the WFS measurements at each time step, it is straightforward to implement a closed loop feedback using a simple integrator: we compute the shape of the deformable mirror based on the previous measurements (multiplied by a loop gain), which we add to the previous commands, and subtract this shape to our turbulent phase screen. This allows us to correct the turbulent phase, while at the same time adding our random command vector $\mathbf{c}_{\xi}$. This closed loop approach, described in Equation 12, effectively applies a high pass filter by using the differential commands $\Delta \mathbf{c}_{t}=\mathbf{c}_{t+1}-\mathbf{c}_{t}$ (of which the turbulence component will be small, since turbulence evolves more slowly than the sampling frequency of the AO system). However, modulating the deformable mirror will inevitably degrade the PSF at the focal plane. We therefore wanted to determine the acceptable level of modulation to still generate valid control matrices and the impact of this modulation level on the Strehl ratio of the delivered PSF. The motivation was to determine whether the DO-CRIME process could be permanently running in the background during observations, providing constant feedback to misalignments and updating optimal control matrices.

To keep things manageable, we decided to neglect noise in these simulations, where we kept the same AO configuration, using the 211 actuator ASM on the UH2,2m telescope with a $16 \times 16$ SH WFS operating at $600 \mathrm{~Hz}$. We first ran the simulation with no feedback and no modulation, but recorded the long exposure PSF: the delivered Strehl ratio on the PSF in open loop was $1.1 \%$ and $0.2 \%$ at $500 \mathrm{~nm}$ and $27.2 \%$ and $9.1 \%$ at $2.2 \mu \mathrm{m}$ for $r_{0}(500 \mathrm{~nm})=0.2 \mathrm{~m}$ and $0.1 \mathrm{~m}$ respectively. Closing the feedback loop with a gain of 0.6 (and a control matrix obtained by inverting a poke matrix), the corrected PSF Strehl ratio was $70.7 \%$ and $32.9 \%$ at $500 \mathrm{~nm}$ and $98.2 \%$ and $94.3 \%$ at $2.2 \mu \mathrm{m}$ for $r_{0}(500 \mathrm{~nm})=0.2 \mathrm{~m}$ and $0.1 \mathrm{~m}$ respectively. Note that these high values are simply due to the very high order system on a $2.2 \mathrm{~m}$ telescope for which $D / r_{0} \sim 2$ at $\mathrm{K}$ band.

We tested modulations of the random command vectors with amplitudes of $0.05,0.1,0.2,0.5,1.0$ and $2.0 \mathrm{~V}$; the corresponding PSFs had Strehl ratios shown in Fig. 8 (top). The results of variance attenuation $\Upsilon_{\sigma_{\epsilon}^{2}}$ are shown on the bottom panel of Fig. 8. As expected, as the modulation amplitude $\mathrm{V}$ becomes larger, the fidelity becomes better and just like in the open loop case, there is an improvement in going from 4000 to 8000 steps, but the performance does not seem to improve by further increasing the number of steps to 16000 or 32000 . Also not completely unexpected, if the modulation is negligible on the Strehl (values of the modulation of 0.05 $-0.5 \mathrm{~V}$ ), the control matrix is degraded with respect to the poke matrix. But as the control matrices improve (modulation range of $1 \sim 2 \mathrm{~V}$ ), it is at the expense of a non-negligible drop of the Strehl ratio in the corrected PSF. In fact a modulation of $2 \mathrm{~V}$ is required to match the poke matrix performance, for which the Strehl ratio is $1.4 \%$ and $0.9 \%$ at $500 \mathrm{~nm}$ and $79.1 \%$ and $76.5 \%$ at $2.2 \mu \mathrm{m}$ for $r_{0}=0.2$ and $0.1 \mathrm{~m}$ respectively.

This is allows to conclude that while it is possible to continually monitor interaction matrices using the DO-CRIME method in closed loop in the background in GLAO mode in the near infrared, the drop in performance is not really compatible with SCAO especially in the visible or in ExAO applications. In this case it may in fact be preferable to measure the interaction matrices between science exposures during camera readouts or filter switches, which is possible because the process is quite fast $(8000$ iterations@600Hz, $\sim 13$ seconds) and does not require any modification in setup or hardware. 

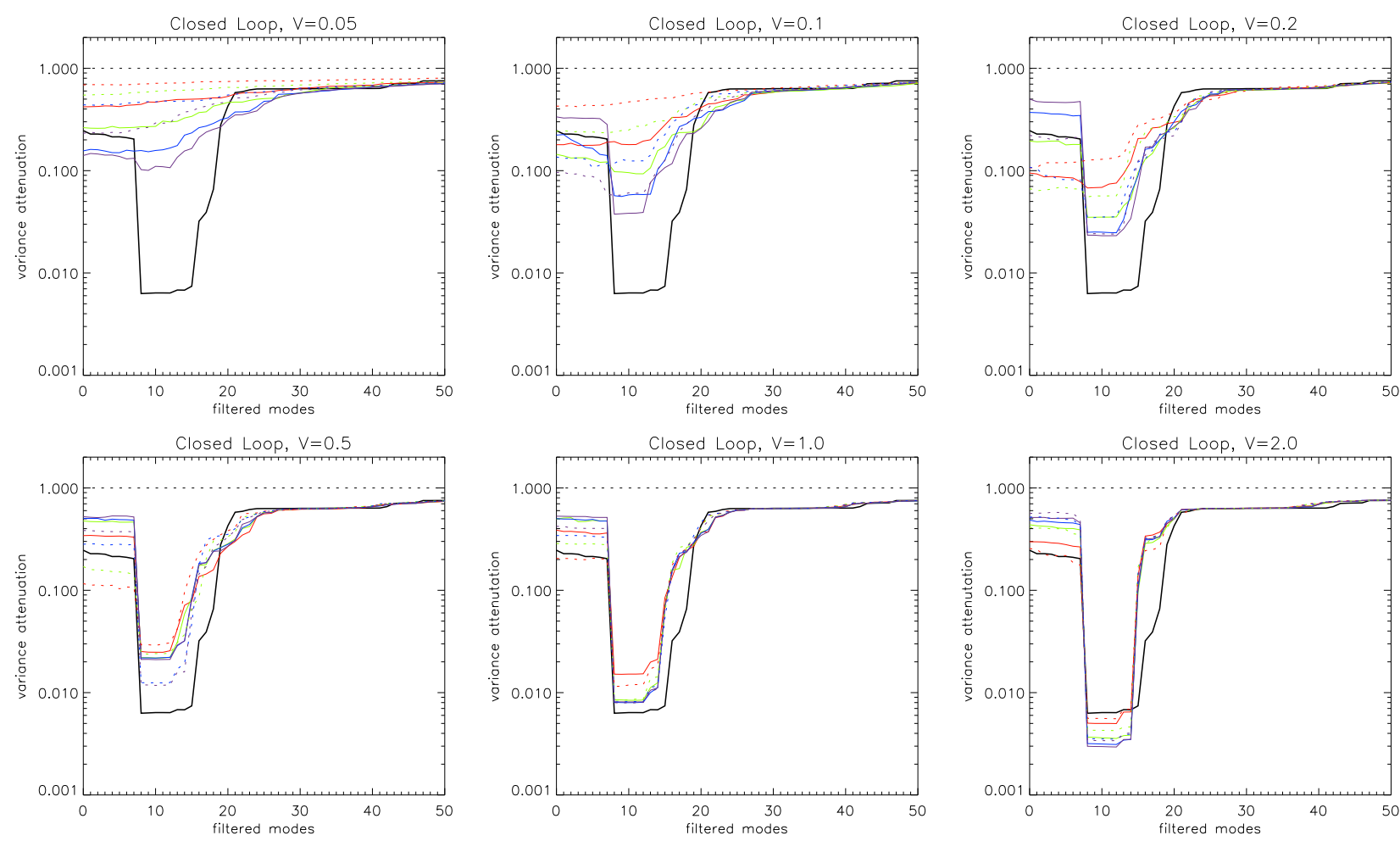

Figure 7. Variance attenuation $\Upsilon_{\sigma_{\epsilon}^{2}}$ as a function of filtered number of modes $\Xi$ for interaction matrices obtained in closed loop with different levels of modulation on the DO-CRIME random vector; black is the poke matrix, red is 4000 iterations, green is 8000 , blue 16000 and purple is 32000 iterations; full line is for $r_{0}=20 \mathrm{~cm}$, dashed line for $r_{0}=10 \mathrm{~cm}$. The larger the modulation, the better (lower) the attenuation, but at the expense of decreasing Strehl ratio at the focal plane PSF (see Fig. 8).

\section{ON-SKY VALIDATION WITH THE 'IMAKA GLAO DEMONSTRATOR}

\subsection{Imaka GLAO demonstrator}

'Imaka is a ground layer adaptive optics (GLAO) demonstrator currently deployed at the UH2,2m telescope (Lai et al. 2008; Chun et al. 2018). It uses five Shack-Hartmann wavefront sensors over a $24^{\prime} \times 18^{\prime}$ field of view, each with $8 \times 8$ subapertures, to control a 36 element bimorph mirror; a description of the performance of 'imaka can be found in Abdurrahman et al. (2018). The geometry of the 'imaka system is shown on Fig. 9. An asymmetric Offner optical relay is used to re-image the pupil on the deformable mirror, but the system will soon be upgraded with an ASM developed by TNO to demonstrate the use of a new type of actuator based on the principle of magnetic reluctance. The use of an ASM on this Cassegrain telescope motivated the development of the DO-CRIME method, but the current optical relay provides an entrance focus which allows to confirm our proof-of-concept experimentally with this system by allowing a direct comparison of DO-CRIME matrices and poke matrices, obtained using calibration sources. Unfortunately, 'imaka interaction matrices are not ideal for this because in a hybrid system using Shack-Hartmann WFS with a bimorph mirror, outer ring adjacent electrodes generate almost identical measurement vectors, leading to poorly conditioned matrices and a certain level of degeneracy on the measurements for different actuators (see Fig. 9, right).

\subsection{Laboratory tests using a single electrode}

Before going on-sky where subtle differences in performance may be hidden by uncorrected free atmosphere turbulence, we performed experiments with the 'imaka AO system using artificial sources in a controlled laboratory setting. This means that the DO-CRIME matrices are obtained without the on-sky background turbulence, but the simulations have shown that we should be able to efficiently reject the atmospheric signal to extract the interaction matrices. The test signal $\mathbf{c}_{a}$ was the modulation of a single actuator (number 13) on the deformable mirror with a sine wave with a period of 32 time steps (at $180 \mathrm{~Hz}$ ) of amplitude $0.2 \mathrm{~V}$ for which we recorded the corresponding measurements $\mathbf{m}_{a}$ on the wavefront sensors. We reconstructed the command vector $D^{+} \mathbf{m}$ and compared it to the input command vector, shown on Fig. 10. Modulating a single electrode is obviously not representative of atmospheric turbulence, but it does allow to see directly the level of crosstalk between electrodes in the reconstruction of each control matrix. We computed the reconstructed commands using different control matrices:

- A poke matrix obtained that day, using the average of 25 measurements poked positively and negatively 3 times for each actuator; 7 modes were filtered at inversion.

- A poke matrix obtained in a similar fashion in January 2018, one year prior.

- An open loop DO-CRIME matrix using a uniform random distribution with commands between $\pm 0.2 \mathrm{~V}$ and 27000 time steps. This required filtering 13 modes at inversion.

- A DO-CRIME matrix obtained in closed loop with the same 


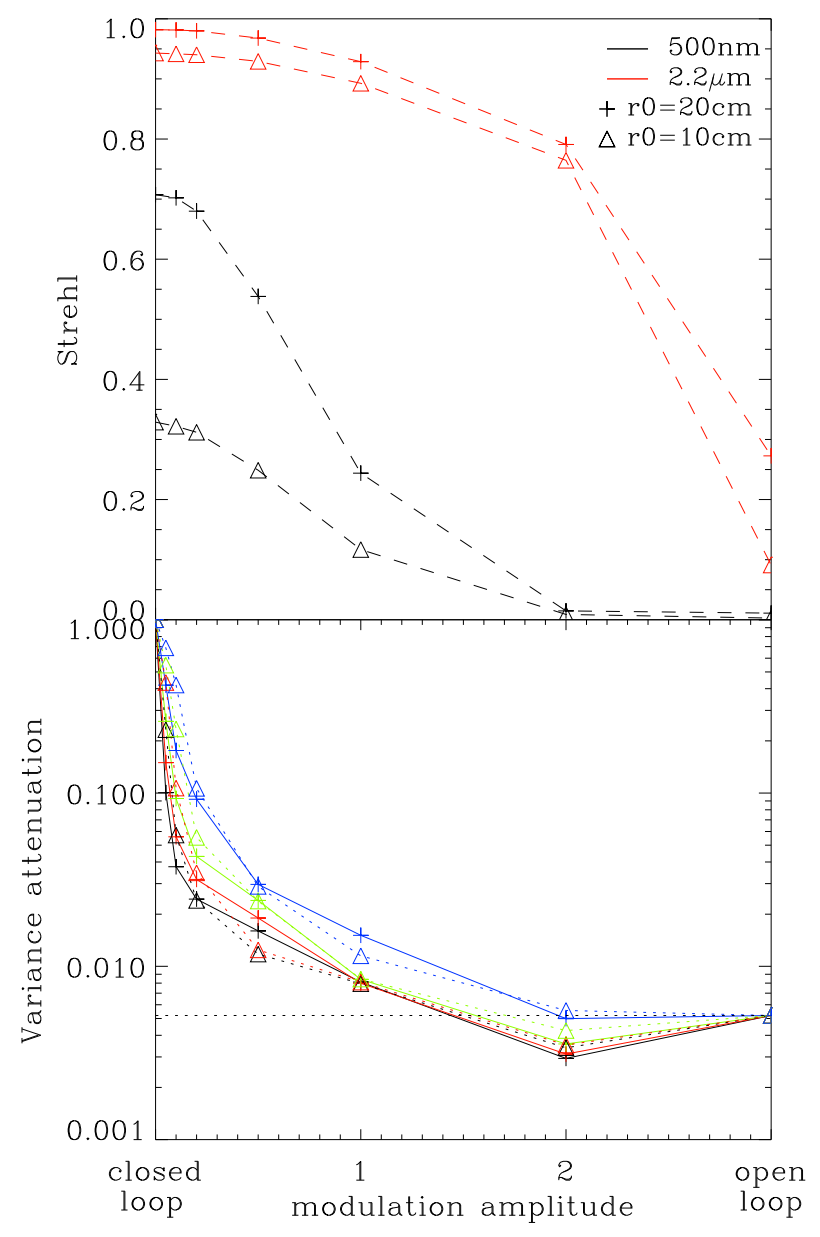

Figure 8. Top: Strehl ratio attenuation (black: $500 \mathrm{~nm}$, red: $2.2 \mu \mathrm{m}$ ) due to modulation on DM; bottom, minimum values of $\Upsilon_{\sigma_{\epsilon}^{2}}$ for optimally filtered number of modes at inversion (blue: 4000, green: 8000 , red: 16000 , black: 32000 iterations, full line: $r_{0}=0.2 \mathrm{~m}$, dashed line: $r_{0}=0.1 \mathrm{~m}$ ). Closed loop (no modulation) with poke matrix is shown for modulation amplitude of zero, and open loop (no modulation, no correction) is shown for modulation amplitude of 3. The poke matrix variance attenuation is shown as the black dashed line.

uniform random distribution command vector, also requiring 13 filtered modes at inversion.

- A DO-CRIME matrix obtained with commands of +0.2 and $-0.2 \mathrm{~V}$ randomly applied on each actuator in open loop, 13 modes filtered.

- And a DO-CRIME matrix obtained in closed loop with the same binary command vector and same number of filtered modes.

The modulation of electrode 13 is well reconstructed by all matrices, except the year old one, implying that the system evolved in some way over the course of this time. The open loop, binary matrix seems to minimize the amount of cross-talk, probably because it maximizes the amount of signal in the $\Delta \mathbf{c}_{\xi}$ vector to compute the interaction matrix.

\subsection{On-sky DO-CRIME matrices}

During an observing run in January 2020, we obtained an interaction matrix using the poke method by pushing and pulling on the actuators with a given voltage ( \pm 0.2 out of a scale of \pm 1 ) multiple times and averaging the displacement of the wavefront sensor spots using a regular centroid algorithm. We then applied different sets of random command vectors $\mathbf{c}_{\xi}$ on sky using natural reference guide stars and recorded the wavefront sensor measurements in open, but also in closed loop (using the control matrix obtained from the poke interaction matrix). We implemented the high pass temporal filter described above on the measurements (and the commands in closed loop), since most of the turbulence occurs at low temporal frequencies, and this improved the signal on the interaction matrices; we found no difference between a Wiener filter (using open loop centroid measurements as input), and a sine squared high pass filter as long as the high temporal frequency content remained unaltered. The different types of random command vectors $\mathbf{c}_{a}$ that we tested on-sky included multiple simultaneous poke commands, random Zernike modes, and uniform random distributions of commands between -0.2 and 0.2 .

We used these measurements to generate control matrices according to equations 10,12 and 7 . We show the interaction matrices, as well as the difference between the on-sky matrices and the poke matrix, on Fig. 11. For clarity, we only display the interaction matrices obtained using equation 12 in open loop, to avoid confusing or unconnected effects linked to matrix inversion or filtering.

These on-sky matrices were obtained with only 4096 samples and non negligible noise which partially explains why these matrices are noisier than the poke matrices. Nonetheless we found that the interaction matrices that showed the least amount of difference with the poke interaction matrix were the ones using random command vectors. The reason for this is that they provide the most diverse set of $\Delta \mathbf{c}$, and thus generate the most varied response on the WFS. As this matrix has a diagonal covariance matrices $\left\langle\Delta \mathbf{c}_{t} \cdot \Delta \mathbf{c}_{t}^{T}\right\rangle$, it is well conditioned and the inversion is straightforward and accurate. On the contrary, the commands covariance matrix for Zernike modes has non-diagonal terms, especially in the outer ring of actuators, which requires more filtering at inversion. This can be seen in the residuals on the third row of Fig. 11. The multiple simultaneous poke commands simply did not provide enough separate occurrences of non-zero $\Delta \mathbf{c}$ to give a strong signal, visible especially on the outer ring actuators (second row of Fig. 11). Finally, the horizontal line at actuator 10 on the difference plot is an example of the advantage of using the DO-CRIME method to incorporate the dynamic behavior of the AO system in the interaction matrices; we discuss this in the next section.

\subsection{Electrode 10}

While measuring the temporal transfer functions of imaka, the dynamical response of electrode number 10 was found to be unreliable (Fig. 12). The reason for this is currently under investigation but could be due to a poor connection of the electrode at the bimorph mirror, which could change its capacitance. However, because the DO-CRIME on-sky matrices are obtained dynamically, we see this row appear in the residuals column of Fig. 11, as a marked difference between the static poke matrices and the on-sky matrices. At inversion, this row is automatically set to zero by SVD inversion and electrode 10 is thus filtered from the command matrix because its dynamic response is not sufficiently linear. In fact further laboratory testing showed that electrode 4 also has a slightly poorer dynamic response, although not at the same level as electrode 10 , so it is usually left in the control matrix at inversion. This illustrates 


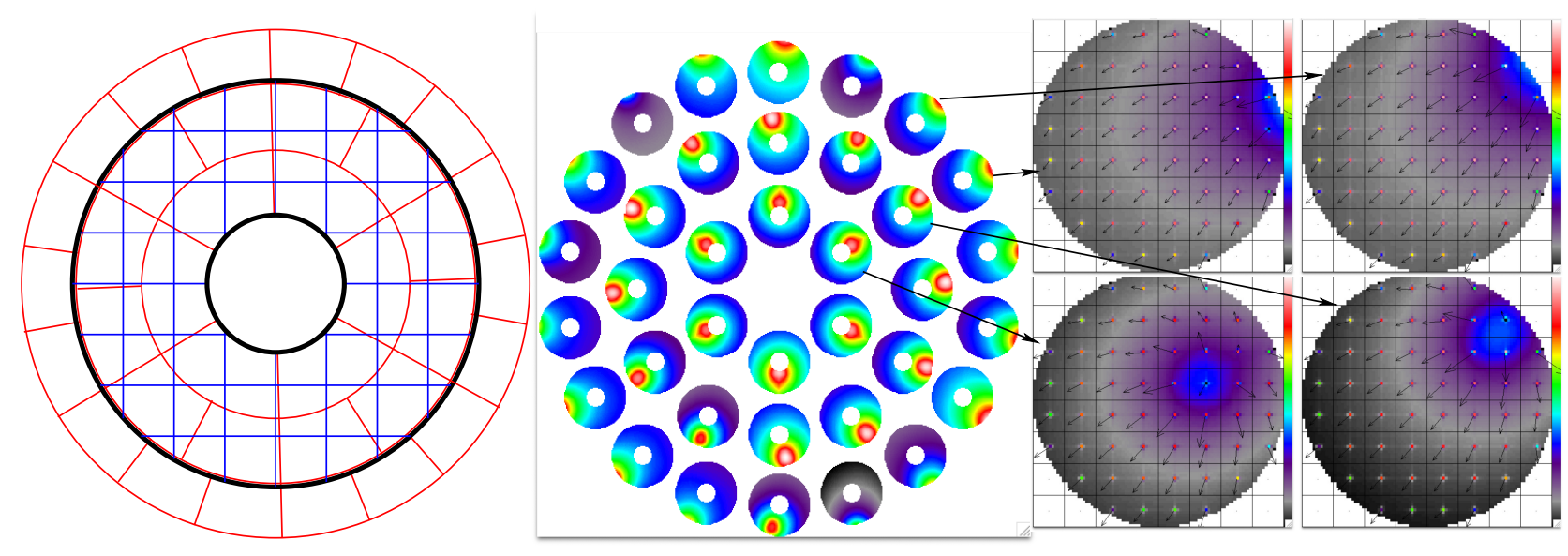

Figure 9. Left: the geometry of the $8 \times 8$ Shack-Hartman wavefront sensor (blue), the bimorph mirror electrodes (red) and the pupil (black think lines). In the middle, the bimorph influence functions are shown as a function of their position in the pupil. Right, the response on the Shack Hartman for electrodes 5 , 16, 32 and 33 is shown, with Shack Hartman images overlaid to the phase and centroid vectors; note how similar electrodes 32 and 33 (top row) appear to the sensor. The Shack Hartman spots are limited by the diffraction of the subapertures.
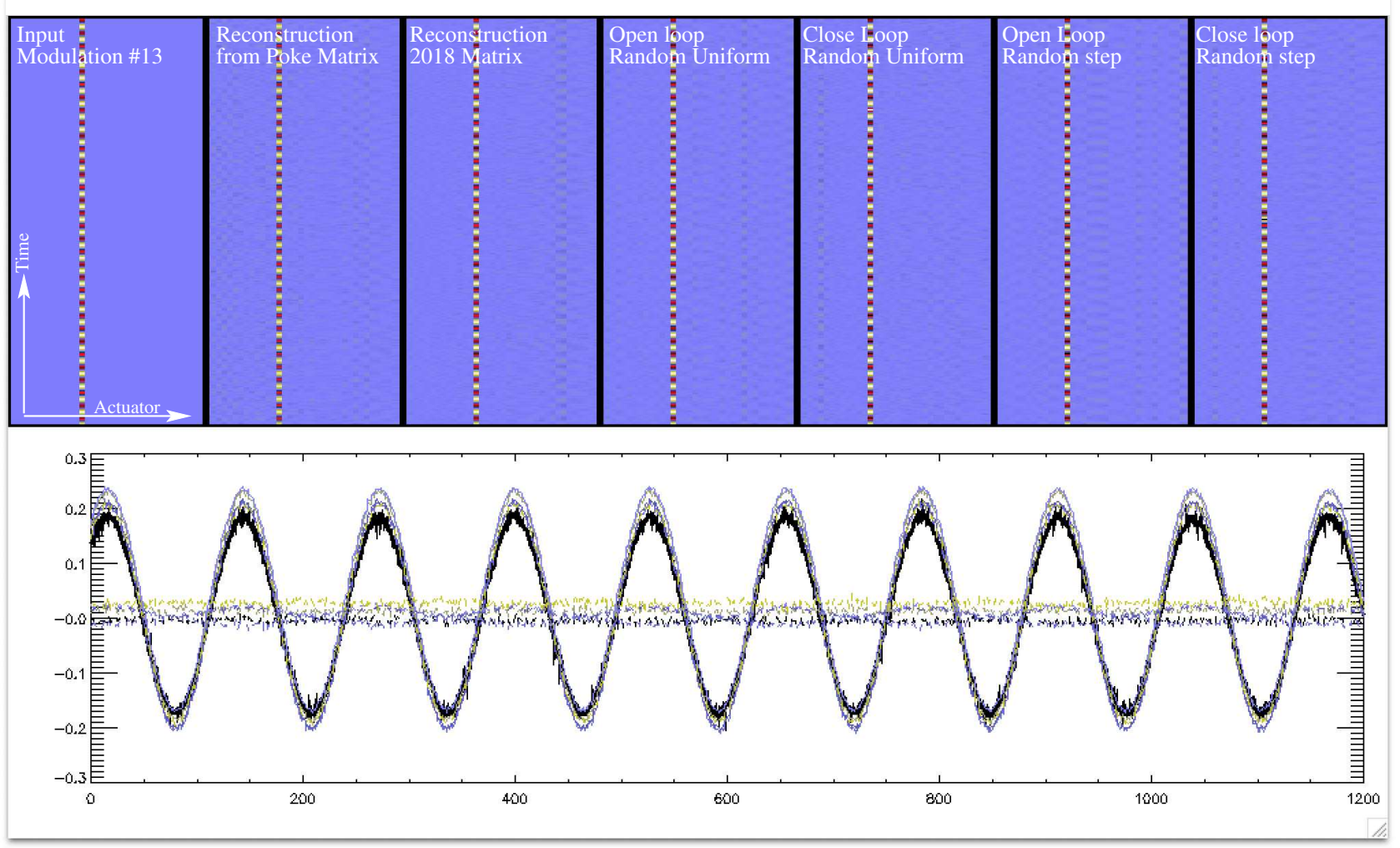

Figure 10. Known signal reconstruction through different control matrices: electrode 13 is modulated with a 32 time step period (top left panel), while all the other actuators are set to zero and measurements are recorded on the wavefront sensor. The next 6 top panels show the reconstructed commands obtained by $\boldsymbol{D}$.m, from left to right: poke matrix, old poke matrix (from 2018, showing that the system evolved) and four different DO-CRIME matrices, open loop using a uniform random distribution, closed loop random distrubution, open loop random step functions and closed loop random step functions. for each of the DO-CRIME matrices, 13 modes had to be filtered at inversion to minimze the cross talk, compared to 7 filtered modes for the poke matrices. The bottom panel shows electrode 13's reconstructed modulation, as well as electrode 32 (outer ring), to give an idea of the scale of the cross talk.

the advantage of measuring interaction matrices in the exact same conditions as those in which the system operates.

\subsection{On-sky validation}

Finally we validated the proof of concept for on-sky matrices using the imaka GLAO system: we compared the FWHM of focal plane images obtained with GLAO correction using poke matrices and two DO-CRIME matrices, obtained by combining the same sequence 


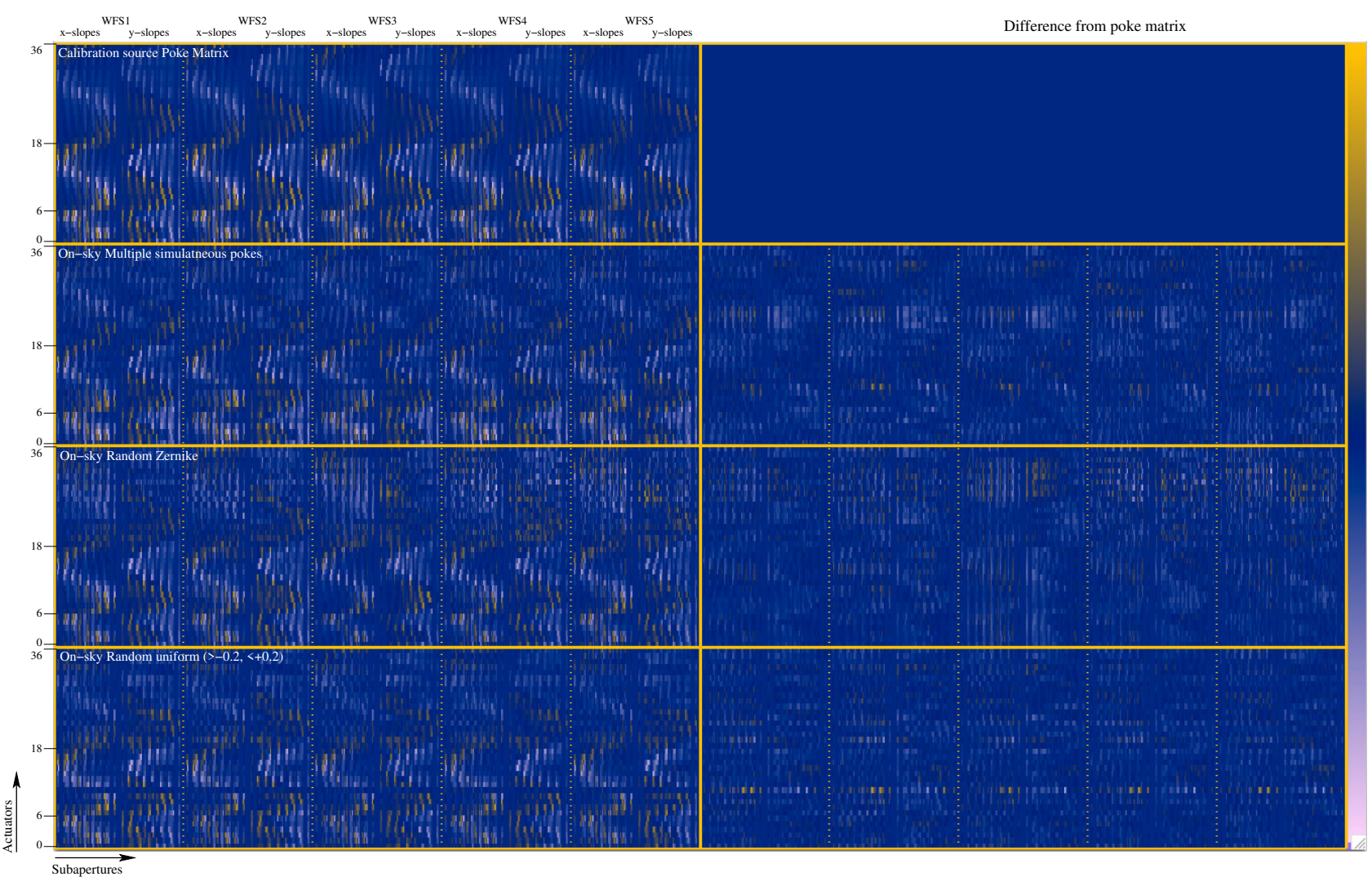

Figure 11. On-sky interaction matrices for imaka, using $5 \mathrm{SH}$ wavefront sensors and a bimorph mirror. The left column shows the matrices, while the right column shows the residuals between the left column and the poke matrix obtained with the calibration source, which is shown on the top row. The second row shows an on-sky matrix obtained with simultaneous multiple ( 2 or 3 at each time step) random poke commands. The next row shows the interaction matrix obtained on-sky using random Zernike commands, while the bottom row shows an interaction matrix obtained with a uniform random distribution of commands between -0.2 and +0.2 . Note how the measurements for the outer actuators are more noisy for the Zernike case, due to poor conditioning of the commands covariance matrix. Also note the line in the residuals at actuator 10 for the random distribution of commands matrices. See text for explanation.
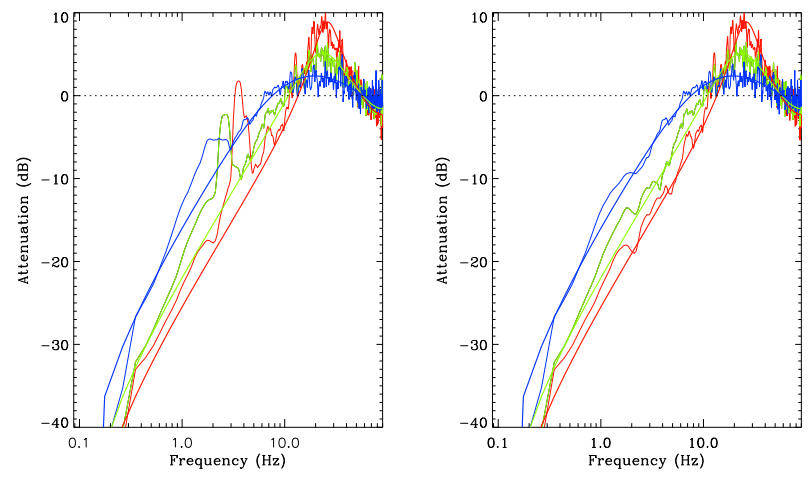

Figure 12. Error transfer functions for imaka with gains of 0.6 (red), 0.4 (green) and 0.1 (blue). The data is obtained in closed loop on an artificial source and no disturbance (the signal is photon noise). The thick line is a model based on the Fourier transform of a simple integrator discrete step function with pure delay of one frame. The left panel shows the error transfer function using all the actuators and the right panel shows exactly the same data but removing actuator 10 from the analysis.

of 4096 random commands uniformly distributed between $-0.2 \mathrm{~V}$ and $+0.2 \mathrm{~V}$ on sky four times. We obtained five sequences where we cycled through open loop, poke matrices and so-called DO-
CRIME 3 and DO-CRIME 4. The results are shown on Table 1 and in Fig. 13; because the residuals are dominated by the uncorrected free atmosphere, subtle reconstruction effects can easily be drowned out, and all matrices provide the same level of performance within error bars. During the 5 sequences, DO-CRIME 3 provided better performance than poke matrices (trial 2 and 5, Fig. 13, left), similar performance (trial 1 and 4) and slightly worse performance (trial 2), while DO-CRIME 4 seems to always have a larger standard deviation in FWHM. It is thus very hard to distinguish the relative performance of these matrices, as those subtle differences can be caused by variations in seeing during our measurements: the open loop FWHM histogram is double peaked (and relatively broad 0.1 "), implying that the atmosphere was not stationary with different regimes at different times and could easily have varied by more than 0.1 " (mean) during our acquisition sequence.

It is unfortunate that the Shack-Hartman-bimorph hybrid configuration generates invisible modes and that the interaction matrices are poorly conditioned, as it makes the performance more sensitive to noise and turbulence and thus it is difficult to assess how effective the control matrices are. Nonetheless, the on-sky performance of DO-CRIME matrices obtained on-sky is comparable to that of poke matrices, at least within error bars. This is comforting as it ensures that we will be able to control the planned ASM for our Cassegrain telescope once it is delivered. 
GLAO FWHM with different cmats (5 sequences)
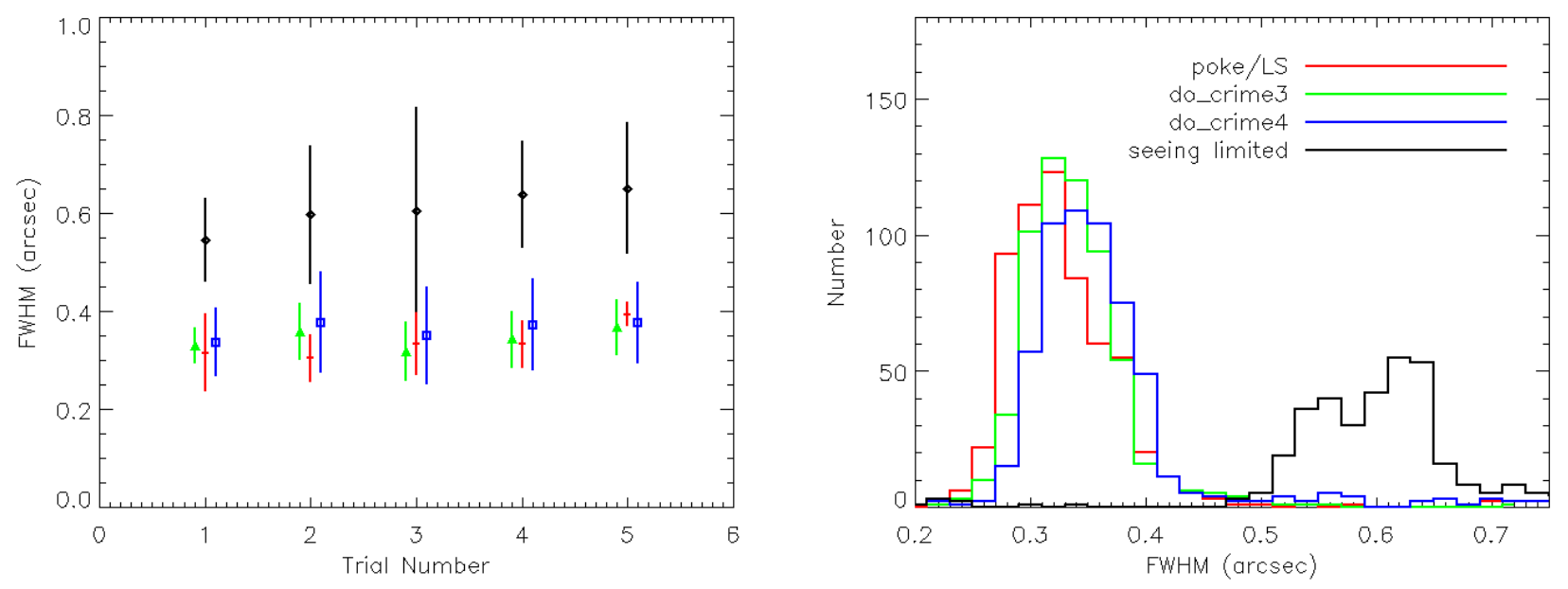

Figure 13. Average and standard deviation for 5 on-sky sequences (left) and histogram (right) of FWHM obtained from on-sky focal plane images. Open loop data is shown in black; the double-peaked histogram suggests that the atmosphere was not stationary during these measurements. Poke matrices are shown red and the DO-CRIME matrices (shown in green for DO-CRIME3 and blue for DO-CRIME4) provided an essentially equivalent level of performance: The DO-CRIME3 matrices sometimes marginally outperform the poke matrices (trial 2 and 5), although the DO-CRIME4 matrices seem to have a systematically larger standard deviation. Within error bars and intrinsic uncertainty due to seeing fluctuations, the DO-CRIME matrices provide a comparable level of performance to the poke matrices.

Table 1. FWHM obtained from focal plane images.

\begin{tabular}{lcc} 
FWHM & Median(") & stdev(") \\
\hline Poke & 0.321 & 0.12 \\
docrime3 & 0.334 & 0.12 \\
docrime4 & 0.345 & 0.14 \\
Seeing & 0.593 & 0.25
\end{tabular}

\section{CONCLUSIONS}

We have presented a novel method for measuring interaction matrices on sky, when no intermediate focus for a calibration source exists, such as with convex adaptive secondary mirrors. The method consists in modulating the deformable mirror using a random (but known) sequence of commands and measuring the associated simultaneous wavefront sensor measurements. Using the property of the modulation covariance matrix being diagonal, thus trivial to invert, we can obtain interaction matrices by multiplying the crosscovariance of the measurements and commands by the inverse of the commands covariance matrix. We have tested the method by simulation using turbulent phase screens, finite element models of the 211 actuator ASM influence functions and a model of our wavefront sensor, both in open loop, where we investigated the impact of measurement noise, as well as the use of high pass filtering to better separate the modulation from the turbulent signal, and in closed loop. In this latter case, we recorded the delivered Strehl ratio at the PSF focal plane to determine an acceptable level of modulation for background operation. We find that in the GLAO regime, modulation at levels much smaller than the residual phase error still allow us to generate acceptable interaction matrices. For SCAO and ExAO, this method is incompatible with the requirements on the accuracy of the control matrix and the level of image degradation in the focal plane. In that case it is therefore probably preferable to measure interaction matrix between science exposures. The need for a constantly updated control matrix is not obvious for most science cases performance, and since adaptive optics systems operate in closed loop, as long as unstable modes are filtered out, errors in the control matrix translate in slightly delayed responses; apart from at visible wavelengths or in cases of exquisite PSF stability, most adaptive optics programs can tolerate slightly suboptimal control matrices, which also explains why they are often neglected in practice. Our method differs slightly from other methods found in the literature in that it is invasive, but very fast, unlike other invasive methods, but it is also completely model independent unlike non-invasive methods.

Nonetheless, we have shown that there are advantages to measuring interaction matrices in the same exact conditions dynamic conditions than those in which they will be used. For example, our method allows us to confirm that electrode \#10 on the bimorph mirror was misbehaving due to a probable bad connection on its connector, slowing down its response. It still showed up in the poke interaction matrix, but was degrading performance when dynamically excited. The DO-CRIME method naturally filters it out, as well as applies a gain to other actuators which may also have non-optimal response. Other examples of benefits to measuring the interaction matrices on sky include accurate treatment of vignetting and diffraction of edge subapertures (which will be filtered out if they become non linear), signal to noise ratio of each subaperture included in the control matrix, centroid gain in the case of quad cell sensors, etc.

We have demonstrated that our method works on sky using the imaka GLAO instrument at the UH2,2m telescope, by generating control matrices using different types of modulation commands (uniform, stepwise, modal) and were able to close the GLAO loop on matrices acquired on sky. This confirms that our method will allow us to control our convex adaptive secondary mirror as soon as it is installed on the telescope. Tools to interpret control matrices in terms of parametric behavior (centering, rotation, magnification, distortion, vignetting) have also been developed for the integration of the ASM, in order to take advantage of the capability of the DOCRIME method to generate interaction matrices on the fly. In a first 
step, we will be able to use them as a tool for alignement on-sky, but more generally also as a diagnostic tool providing a physical interpretation of the $\mathrm{AO}$ system state.

\section{ACKNOWLEDGEMENTS}

'imaka is supported by the National Science Foundation under Grant No. AST-1310706 and by the Mount Cuba Astronomical Foundation. We thank Stefan Kuiper (TNO) for providing the model influence functions for the UH88 adaptive secondary mirror. The authors also wish to recognize and acknowledge the very significant cultural role and reverence that the summit of Maunakea has always had within the indigenous Hawaiian community. We are most fortunate to have the opportunity to conduct observations from this mountain.

\section{$U H: 2.2 m:$ ('imaka)}

\section{DATA AVAILABILITY STATEMENT}

The data and simulation code underlying this article will be shared on reasonable request to the corresponding author.

\section{REFERENCES}

Abdurrahman, F. N., Lu, J. R., Chun, M., Service, M. W., Lai, O., Föhring, D., Toomey, D., Baranec, C., 2018, "Improved Image Quality over $10^{\circ}$ Fields with the "Imaka Ground-layer Adaptive Optics Experiment". The Astronomical Journal, 156(3), id.100, 17pp.

Babcock H., 1953, "The Possibility of Compensating Astronomical Seeing". Publications of the Astronomical Society of the Pacific, 65(386), 229.

Bechet, C., Kolb, J., Madec, P.-Y., Tallon, M., Thiébaut, E., "Identification of system misregistration during AO-corrected observations", Proc. AO4ELT2, 2011, http://ao4elt2.lesia.obspm.fr/sites/ao4elt2/IMG/pdf/056bechet.pdf

Bechet, C., Tallon, M., Thiébaut, E., "Optimization of adaptive optics correction during observations: Algorithms and system parameters identification in closed loop", Proc. SPIE Adaptive Optics Systems III, 2012, Vol. 8447, 8447C-1.

Chun, M., Lu, J., Lai, O., Abdurrahman, F., Service, M., Toomey, D., Fohring, D., Baranec, C., Hayano, Y., Oya, S., 2018, "On-sky results from the wide-field ground-layer adaptive optics demonstrator 'imaka". Proc. SPIE Adaptive Optical Systems, 2018. Vol. 10703, p. 7.

Esposito, S., Tubbs,R. , Puglisi, A., Oberti, S., Tozzi, A., Xompero, M., Zanotti, D., âĂIJHigh SNR measurement of interaction matrix on-sky and in labâĂİ Proc. SPIE Advances in Adaptive Optics II, 2006, Vol. 6272, 62721C.

Gaffard J.P., Boyer C., âĂIJAdaptive optics for optimization of image resolutionâĂİ, Applied Optics, Vol 26, p 3772, (1987).

Gendron, E., Léna, P., "Astronomical adaptive optics. I. Modal control optimization", Astron. \& Astrophys., 291, 337-347.

Heritier, C.T., Fusco, T., Neichel, B., Esposito, S., Oberti, S., Correia, C., Sauvage, J.-F., Bond, C., Fauvarque, O., Pinna, E., Agapito, G., Puglisi, A., Kolb, J., Madec, P.-Y., Bechet, C., "Overview of AO calibration strategies in the ELT context". Proc AO4ELT5, 2017, http://research.iac.es/congreso/AO4ELT5/media/proceedings/proceeding035.pdf

Heritier, C.T., Esposito, S., Fusco, T., Neichel, B.,Oberti, S., Briguglio, R., Agapito, G., Puglisi, A., Pinna, E., Madec, P.-Y., "A new calibration strategy for adaptive telescopes with pyramid WFS". Mon. Not. Royal Astron. Soc., 481(2), 2018, 2829-2840.

Heritier, C.T., "Innovative Calibration Strategies for Large Adaptive Telescopes with Pyramid Wave-Front Sensors". Ph.D. thesis Aix Marseille Université, 2019, HAL Id : tel-02390861.
Kasper, M., Fedrigo, E., Looze, D.P., Bonnet, H., Ivanescu, L., Oberti, S., "Fast calibration of high order adaptive optics systems". J. Opt. Soc. Am. A, 21(6), 2004, 1004-1008.

Kolb J., Madec, P.-Y., Le Louarn, M., Muller, N., Béchet, C., "Calibration strategy of the AOF," Proc. SPIE Adaptive Optics Systems III, 2012, Vol. 8447, 84472D.

Meimon, S., Petit, D., Fusco, T., "Optimized calibration strategy for high order adaptive optics systems in closed-loop: the slope-oriented Hadamard actuation," Optics Express, 23(21), 2015, 27134-27144.

Lai, O., Stomski, P., Gendron, E., "MANO: the modal analysis and noise optimization program for the W.M. Keck Observatory adaptive optics system", Proc. SPIE Adaptive Optical Systems Technology Vol. 4007, 2000, 620-631.

Lai, O., Chun, M., Cuillandre, J.-C., Carlberg, R., Richer, H., Andersen, D., . Pazder, J., Tonry, J., Doyon, R., Thibault, S., Dunlop, J., Pritchet, C., Véran, J.P., Ftaclas, C., Onaka, P., Hodapp, K.W., McLaren, R.A.. Bertin, E., Mellier, Y., Astier, P., Pain, R., 2008, "IMAKA: imaging from Mauna KeA with an atmosphere corrected 1 square degree optical imager". Proc SPIE Adaptive Optics Systems, 2008, Vol. 7015, p.12.

Lai, O., Chun, M., Abdurrahman, F., Lu, J., Service, M., Fohring, D.,Toomey, D., "Deconstructing turbulence and optimizing GLAO using imaka telemetry". Proc. SPIE Adaptive Optical Systems, 2018. Vol. 10703, 107036D.

Neichel B., Parisot, A., Petit, C., Fusco, T., Rigaut, F., "Identification and calibration of the interaction matrix parameters for $\mathrm{AO}$ and MCAO systems", Proc. SPIE Advances in Adaptive Optics II, 2006, Vol. 6272, 627220 .

Oberti, S., Quirós-Pacheco, F., Esposito, S., Muradore, R., Arsenault, R., Fedrigo, E., Kasper, M., Kolb, J., Marchetti, E., Riccardi, A., Soenke, C., Stroebele, S., "" Proc. SPIE Adaptive Optics Systems III, 2012, Vol. $8447,84475 \mathrm{~N}$.

Pirealli, F., Puglisi, A., Quiros Pacheco, F., Esposito, S., "Sinusoidal calibration technique for Large Binocular Telescope system," Proc. SPIE Adaptive Optics Systems III, 2008, Vol. 7015, 70153A-7.

Roddier, F., 1988, "Curvature sensing and compensation: a new concept in adaptive optics". Applied Optics 27(7), 1988, 1223-1225.

Roddier, F., 1999, "Adaptive Optics in Astronomy". Cambridge: Cambridge University Press.

Tyson, R.K., 1999, "Principles of Adaptive Optics". Cambridge: Academic Press.

Wildi, F.P., Brusa, G., "Determining the interaction matrix using starlight", Proc. SPIE Advancements in Adaptive Optics, 2004, Vol. 5490, 164173.

Woillez, J., Abad, J., Abuter, R., Carpentier, E., Alonso, J., et al., "NAOMI: the adaptive optics system of the Auxiliary Telescopes of the VLTI. Astronomy and Astrophysics" A\&A, 629, A41 (2019)

This paper has been typeset from a $\mathrm{T}_{\mathrm{E}} \mathrm{X} / \mathrm{L} \mathrm{T}_{\mathrm{E}} \mathrm{X}$ file prepared by the author. 